\title{
Redesign of Marine Structures by Perturbation
}

\author{
Jonghyun Kim \\ $\&$ \\ Michael M. Bernitsas \\ Department of Naval Architecture and Marine Engineering, The University of Michigan, \\ Ann Arbor, Michigan 48109-2145, USA \\ (Received 6 January 1988; revised version received 26 May 1988; accepted 17 June 1988)
}

\begin{abstract}
Finite element (FE) methods are extensively used for analysis of static and dynamic behavior of marine structures. Often predicted response is unacceptable from the point of view of design or operation. Improvement of response then, becomes a design goal which can be achieved by redesign or reduction of operational threshold. Traditional trial and error techniques using FE methods make redesign expensive and are often inconclusive. In this paper a perturbation-based method is developed to solve redesign problems with both static and modal dynamic objectives using data only from the $F E$ analysis of the baseline structure. Code RESTRUCT implements this method and functions as postprocessor to general or special purpose FE codes. Several simple numerical applications are used to illustrate the efficiency of this redesign method and how it can be used to resolve conflicts caused by incompatible redesign requirements. A 192-degree-of-freedom tower with repeated eigenvalues is redesigned subject to frequency and displacement constraints. Finally the impact of perturbation-based redesign on marine structural design is discussed.
\end{abstract}

Key words: Redesign, marine structures, perturbation, static and modal dynamic objectives, finite elements.

Marine Structures 0951-8339/88/\$03.50 1988 Elsevier Science Publishers Ltd, England. Printed in Great Britain. 


\section{NOTATION}

$b_{u_{i^{\prime}}} b_{\omega_{i^{\prime}}}, b_{\phi_{k i}} \quad$ Bounds on $\Delta u_{i}, \Delta \omega_{i}, \Delta \phi_{k i}$, respectively

$c_{i j}$

Admixture coefficient $c_{i j}$; participation of the $j$ th mode to changes in the $i$ th mode

$D_{i}, D_{\mathrm{i}}{ }^{\prime} \quad$ Inner diameter of tubular element of baseline and objective structures

$D_{o}, D_{\mathrm{o}}^{\prime}$

E

$\{f\}$

$f_{j}$

$[k],\left[k^{\prime}\right]$

Outer diameter of tubular element of baseline and objective structures

$\left[k_{e}\right],\left[k_{e}^{\prime}\right]$

Young's modulus

Nodal force vector

$j$ th component of vector $\{f\}$

Stiffness matrix of baseline and objective structures

Baseline and objective stiffness matrices of element or group of elements related to property $e$

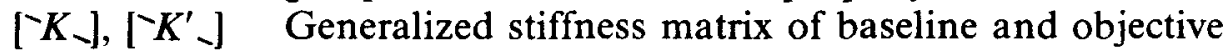
structures

$l$

$[m],\left[m^{\prime}\right]$

Index denoting quantities in increment $l$

$\left[m_{e}\right],\left[m_{e}^{\prime}\right]$

Mass matrix of baseline and objective structures

$M_{i}, M_{i}^{\prime}$

Baseline and objective mass matrix of the element or group of elements related to property $e$

['M ], [-M']

Generalized mass of the $i$ th mode of baseline and objective structures

['M $],\left[-M^{\prime}\right]$

Generalized mass matrix of baseline and objective structures

$n$
$n_{\mathrm{r}}$
$N$

Number of degrees of freedom in structural model

Number of modes of the baseline structure used in redesign

Total number of increments in predictor-corrector algorithm

$p$

Number of design variables

$q$

$\left\{Q^{\prime}\right\}$

$r_{u}, r_{\omega}, r_{\phi}$

$S$

$S_{\mathrm{b}}$

$S_{\mathrm{e}}$

$S_{\mathrm{i}}$

$S_{u}$

$S_{\omega}$

Increment number in predictor-corrector algorithm; $q=1,2, \ldots, N$.

Transformed displacement vector

Incremental changes defined by eqns (34), (35), (36)

Total number of redesign constraints

Total number of upper and lower bound inequality constraints imposed on $\alpha_{e} s$

Number of equality redesign constraints

Number of inequality redesign constraints

Number of displacement redesign constraints

Number of frequency redesign constraints 

$S_{\phi}$
$\{u\},\left\{u^{\prime}\right\}$
[]$^{\mathrm{T}},\{\}^{\mathrm{T}}$
[]$^{-1}$
Number of modal node redesign constraints
Nodal displacement vector of baseline and objective structures
Denote transpose of a matrix and vector, respectively
Denotes inverse of a square matrix
Denotes a diagonal matrix

Greek symbols

$a_{e}$

$\Delta$

$[\Delta k]$

$\left[\Delta k_{e}\right]$

$[\Delta m]$

$\left[\Delta m_{e}\right]$

$\{\Delta \mathrm{u}\}$

$\{\Delta \phi\}$

$\Delta\left(\omega_{i}^{2}\right)$

$[\phi],\left[\phi^{\prime}\right]$

$\{\psi\}_{i},\left\{\psi^{\prime}\right\}_{i}$

$\phi_{k i}$

$\omega_{i}, \omega_{i}^{\prime}$
Fractional change to element or group property $e$

Prefix denoting incremental change to structural quantities Prefix denoting total change to structural quantities

Change to stiffness matrix $[k]$

Change to matrix $\left[k_{e}\right]$

Change to mass matrix $[m]$

Change to matrix $\left[m_{e}\right]$

Change to the nodal displacement vector

Matrix of the mode shape vector changes

Change to the $i$ th eigenvalue

Matrix of mode shape vectors of baseline and objective structures

$i$ th mode shape of baseline and objective structures

$k$ th degree of freedom of $i$ th baseline mode shape

$i$ th natural frequency of baseline and objective structures

\section{INTRODUCTION}

Perturbation-based redesign methods were first developed in linear form by Stetson $e t$ al.$^{1-4}$ and were further improved by Sandström et al..$^{5,6}$ Linear perturbation methods are applicable to problems where small structural changes are allowed and small changes in undesirable response particulars (static or modal dynamic) are adequate. Nonlinear perturbation methods allowing for large changes in response particulars and structural properties were developed by Kim et al.$^{7-9}$ and Bernitsas, Hoff et al. ${ }^{10-17} \mathrm{Kim}$ et al. ${ }^{7}$ have used a penalty method to satisfy modal dynamic objectives in the redesign process. This method is theoretically exact but a desired solution can be achieved only for small-scale structures due to inaccuracy of the nonlinear numerical search technique used. For larger scale structures they used dynamic condensation which introduces an additional source of numerical error but reduces the prohibitively high cost of the standard methods. ${ }^{8,9}$ In previous work the authors have developed algorithms for solution of the nonlinear perturbation redesign 
problem for only static $^{16}$ or only modal dynamic ${ }^{11-14}$ objectives. Extensive reviews and comparison of perturbation methods are provided in Ref. 13. The model developed in Ref. 12 was recently extended by Gans ${ }^{18}$ to include centrifugal and Coriolis effects. In this work a nonlinear perturbation redesign algorithm is developed for solving redesign problems with simultaneous static and modal dynamic objectives.

Perturbation-based redesign does not produce globally optimal design. Actually redesign problems may or may not be formulated as optimization problems depending on the number of constraints and allowable changes (see Section 4). If a redesign problem results in an optimization problem, computer code RESTRUCT (REdesign of STRUCTures), which implements the algorithm developed in this work, will produce an optimal design in the vicinity of the baseline structure and within the domain defined by the redesign constraints (design goals). In that sense the method developed in this work is related to another type of research, namely design optimization based on sensitivity. ${ }^{19-21}$ These methods produce optimal design. They are efficient when analytical expressions of the sensitivity parameters are available. These parameters are valid within small structural changes. The perturbation-based redesign methods developed in this work are not limited by the size of the structural changes. The problem of repeated eigenvalues can also be handled in this work, as illustrated by the offshore tower application.

In the analysis and design process, diagrammatically shown in Fig. 1, the designer usually generates a FE model for his structure and runs a general or special purpose FE program to analyze the structure's static and/or dynamic response. After identifying possible undesirable static and/or dynamic response characteristics - e.g. vibrations unfavorable to fatigue life, large amplitude vibrations, large static stresses - he has to develop a strategy for modifying his baseline structure in order to eliminate or alleviate such undesirable response. Thus he defines the goals of his design effort which become constraints in the redesign process. The method developed in this work allows the designer to modify modal properties and/or static displacements to achieve his design goals. That is, this method allows the designer to place constraints on displacement and modal properties in redesign. For this he has to specify which substructures or elements may be allowed to change. RESTRUCT will produce an objective design, if one exists. If the designer's goals, that is the redesign constraints, are incompatible, an objective design will not exist. The designer may then accept the solution produced by RESTRUCT which compromises conflicting constraints, 


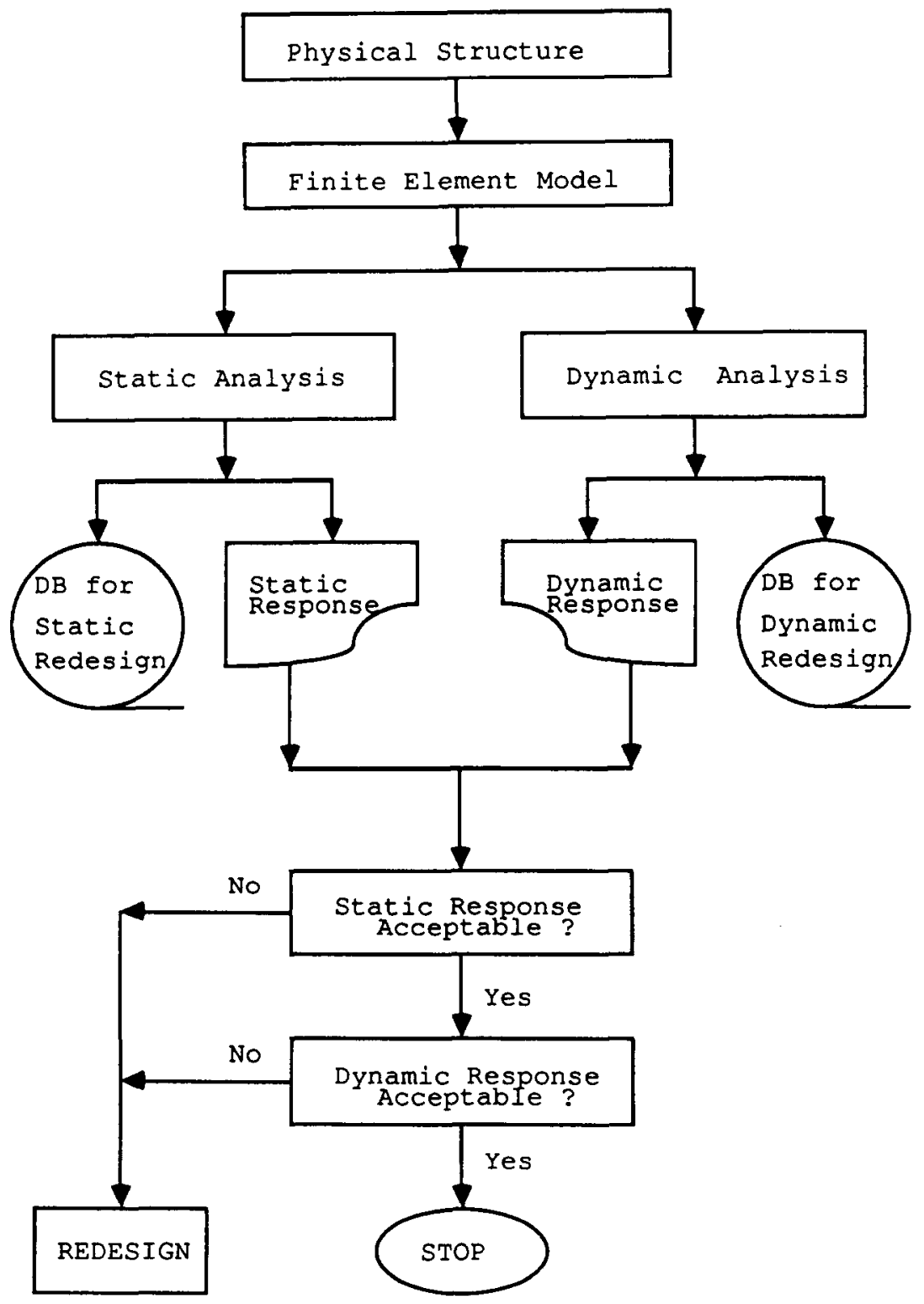

Fig. 1. Block diagram for structural analysis and design.

or he must redefine the redesign problem by changing constraints and/or allowable changes (redesign variables). In any event RESTRUCT uses only results from a single FE program run, that for the baseline structure.

The problem of redesign by perturbation is defined in Section 2 in the general case where the redesign process constraints are both static and 
modal dynamic. These constraints are in general in an implicit nonlinear form and must be modified before they can be used to solve for the unknown structural changes. In Section 3 the redesign algorithm for modification of simultaneously applied static and modal dynamic constraints is developed, and briefly compared to algorithms for static or dynamic redesign developed in previous work. ${ }^{11,14,16}$ In Section 4 the algorithms used for computation of structural changes are explained. Computer implementation of the redesign algorithm is presented in Section 5. The developed computer code serves as postprocessor to FE codes and produces the objective design at a small cost compared to the cost of trial and error methods. Intricacies of redesign related to conflicting design objectives (redesign constraints) and redesign of structures with repeated eigenvalues are illustrated and resolved in Section 6 using numerical applications. In the concluding section the impact of the perturbation-based redesign method developed in this work on marine structural design is assessed.

\section{REDESIGN BY PERTURBATION}

According to the rationale for structural analysis and design shown diagrammatically in Fig. 1, a marine structure is first analyzed by a FE code. Then redesign is performed as necessary using the discretized structural FE model. In this section the problem of redesign of the discretized structure by perturbation is defined. Three different problems can be identified depending on whether design goals - that is redesign constraints - are static, modal dynamic or both. In previous work, as explained in the Introduction, the authors have defined the problems of static $^{16}$ and dynamic ${ }^{11,14}$ redesign by perturbation and developed solution algorithms. In this section the integrated static/dynamic redesign problem is defined.

First the static part of the integrated problem is defined. Let the equation of static equilibrium of the baseline discretized structure be

$$
[k]_{n \times n}\{u\}_{n \times 1}=\{f\}_{n \times 1}
$$

where $[k]$ is the global stiffness matrix, $\{u\}$ is the nodal displacement vector, and $\{f\}$ is the nodal force vector. Equation (1) can be solved for the displacement vector $\{u\}$ which can be used subsequently for computation of stresses. If either static displacements or stresses of this baseline structure violate design constraints the structure must be redesigned. Assume that a structure which satisfies all design constraints - and differs from the basic structure only in structural or material 
properties of elements and not in location of nodes - exists. Let quantities related to this objective structure be primed. Then the governing equation of the objective structure is

$$
\left[k^{\prime}\right]\left\{u^{\prime}\right\}=\{f\}
$$

where $\left[k^{\prime}\right]$ is the objective stiffness matrix, $\left\{u^{\prime}\right\}$ is the objective displacement vector, and the same nodal force vector is assumed as in the baseline model. To relate the baseline and objective models the following perturbation relations are used:

$$
\begin{aligned}
& {\left[k^{\prime}\right]=[k]+[\Delta k]} \\
& \left\{u^{\prime}\right\}=\{u\}+\{\Delta u\}
\end{aligned}
$$

where $[\Delta k]$ is the change in the stiffness matrix, and $[\Delta u]$ is the change in the displacement vector. Applying eqns (3) and (4) to eqn (2) the general static perturbation equation governing the manner in which the structure may change is developed:

$$
[\Delta k]\{u\}+[\Delta k]\{\Delta u\}+[k]\{\Delta u\}=\{0\}
$$

Equation (5) also can be written as

$$
\{\Delta u\}=-([k]+[\Delta k])^{-1}[\Delta k]\{u\}
$$

Further assume that $p$ properties of elements or groups of elements are allowed to change and let $\alpha_{e}$ be the fractional change in property $e$. More than one property may be changed in each element or group of elements. Then expressing the change in the global stiffness matrix as sum of all changes we get

$$
[\Delta k]=\sum_{e=1}^{p}\left[\Delta k_{e}\right]=\sum_{e=1}^{p}\left[k_{e}\right] \alpha_{e}
$$

where several $\left[k_{e}\right] \alpha_{e}$ terms may refer to the same element but different properties like bending, torsion, stretching, etc. For example, for a beam element we may have four change properties; that is, bending in two directions, torsion and axial tension. Inserting in eqn (6) the expression for $[\Delta k]$ from eqn (7) we derive the following expression for the change to displacements between baseline and objective structures:

$$
\{\Delta u\}=-\sum_{e=1}^{p}\left([k]+\sum_{e=1}^{p}\left[k_{e}\right] \alpha_{e}\right)^{-1}\left[k_{e}\right]\{u\} \alpha_{e}
$$

In redesign the designer is allowed to place constraints on displacement changes to achieve his design goals. These may be related directly to 
changes to displacements or indirectly to reduction of stress levels. Selecting out of eqn (8) only those degrees of freedom for which constraints on displacement changes are imposed we get a system of simultaneous nonlinear implicit equations and/or inequalities in $\alpha_{e}$. Due to their complexity they must first be modified using the algorithm developed in Section 3 before they can be used to produce the values of $\alpha_{e}$ s by employing the solvers presented in Section 4 .

Next the dynamic part of the integrated problem is studied. In the redesign process we also allow the designer to change modal characteristics of the baseline structure. Thus, a technique similar to the one presented above for static constraints is used below in order to develop perturbation equations for modal constraints. Let the equation of dynamic equilibrium for the vibration of the baseline discretized structure be

$$
\left([k]-\omega_{j}^{2}[m]\right)\{\psi\}_{j}=\{0\} \text { for } j=1,2 \ldots, n
$$

where $\{\psi\}_{j}$ is the $j$ th mode shape and $\omega_{j}$ is the corresponding natural frequency defined as the $j$ th eigenvalue of eqn (10):

$$
\operatorname{det}\left([k]-\omega^{2}[m]\right)=0
$$

$[m]$ is the $n \times n$ global mass matrix which may include added mass, and damping may be included only in the Rayleigh form by contributing to $[k]$ and $[m]$. Combining eqns (9) into matrix form, we have

$$
[k][\phi]=[m][\phi]\left[-\omega^{2}\right]
$$

where $[\phi]=\left[\{\psi\}_{1},\{\psi\}_{2}, \ldots,\{\psi\}_{n}\right]$, and $\left[-\omega^{2}\right]$ is the diagonal matrix of the eigenvalues. Premultiplying eqn (11) by $[\phi]^{\mathrm{T}}$ we obtain the uncoupled modal equations in the following matrix form:

$$
\left[-K_{-}\right]=\left[-M_{-}\right]\left[-\omega^{2}\right]
$$

where $[K]$ is the generalized stiffness matrix

$$
\left[-K_{-}\right]=\left[K_{i i-}\right]=[\phi]^{\mathrm{T}}[k][\phi]
$$

and $[M]$ is the generalized mass matrix

$$
\left[-M_{-}\right]=\left[M_{i i}\right]=[\phi]^{\mathrm{T}}[m][\phi]
$$

The counterpart equations for the objective structure are

$$
\begin{gathered}
\left(\left[k^{\prime}\right]-\omega_{j}^{\prime 2}\left[m^{\prime}\right]\right)\left\{\psi^{\prime}\right\}_{j}=\{0\} \text { for } j=1,2, \ldots, n \\
\operatorname{det}\left(\left[k^{\prime}\right]-\omega^{\prime 2}\left[m^{\prime}\right]\right)=0 \\
{\left[k^{\prime}\right]\left[\phi^{\prime}\right]=\left[m^{\prime}\right]\left[\phi^{\prime}\right]\left[-\omega^{\prime 2}-\right]} \\
{\left[-K^{\prime},\right]=\left[-M_{-}^{\prime}\left[\left[\omega^{\prime 2}-\right]\right.\right.}
\end{gathered}
$$




$$
\begin{aligned}
{\left[-K^{\prime}-\right] } & =\left[\phi^{\prime}\right]^{\mathrm{T}}\left[k^{\prime}\right]\left[\phi^{\prime}\right] \\
{\left[-M^{\prime}-\right] } & =\left[\phi^{\prime}\right]^{\mathrm{T}}\left[m^{\prime}\right]\left[\phi^{\prime}\right]
\end{aligned}
$$

Further we introduce the following three perturbation relations between the baseline and the objective structure:

$$
\begin{gathered}
{\left[m^{\prime}\right]=[m]+[\Delta m]} \\
{\left[-\omega^{\prime 2}-\right]=\left[\omega^{2}-\right]+\left[-\Delta\left(\omega^{2}\right)-\right]} \\
{\left[\phi^{\prime}\right]=[\phi]+[\Delta \phi]}
\end{gathered}
$$

where $\left.[\Delta m],\left[-\Delta\left(\omega^{2}\right)\right]\right]$ and $[\Delta \phi]$ are changes to mass matrix, eigenvalues and eigenmodes, respectively. Allowing changes to the mass matrix we get the counterpart of eqn (7):

$$
[\Delta m]=\sum_{e=1}^{p}\left[\Delta m_{e}\right]=\sum_{e=1}^{p}\left[m_{e}\right] \alpha_{e}
$$

Inserting eqns (19) and (20) in eqn (18) and using perturbation relations (3) and (21) the general modal perturbation equation is derived as

$$
\begin{aligned}
{\left[\phi^{\prime}\right]^{\mathrm{T}}[\Delta k]\left[\phi^{\prime}\right]-\left[\phi^{\prime}\right]^{\mathrm{T}}[\Delta m]\left[\phi^{\prime}\right]\left[\omega^{\prime 2}\right] } \\
=\left[\phi^{\prime}\right]^{\mathrm{T}}[m]\left[\phi^{\prime}\right]\left[\omega^{\prime 2}-\right]-\left[\phi^{\prime}\right]^{\mathrm{T}}[k]\left[\phi^{\prime}\right]
\end{aligned}
$$

This equation can be decomposed into the following $n^{2}$ scalar equations:

$$
\begin{gathered}
\left\{\psi^{\prime}\right\}_{j}^{\mathrm{T}}[\Delta k]\left\{\psi^{\prime}\right\}_{i}-\omega_{i}^{\prime 2}\left\{\psi^{\prime}\right\}_{j}^{\mathrm{T}}[\Delta m]\left\{\psi^{\prime}\right\}_{i}=\left\{\psi^{\prime}\right\}_{j}^{\mathrm{T}}[m]\left\{\psi^{\prime}\right\}_{i} \omega_{i}^{\prime 2} \\
-\left\{\psi^{\prime}\right\}_{j}^{\mathrm{T}}[k]\left\{\psi^{\prime}\right\}_{i}
\end{gathered}
$$

for $i, j=1,2, \ldots, n$. Equations (26) can be expressed in terms of the structural changes $\alpha_{e}$ s using eqns (7) and (24) as

$$
\begin{aligned}
\sum_{e=1}^{p}\left(\left\{\psi^{\prime}\right\}_{j}^{\mathrm{T}}\left[k_{e}\right]\left\{\psi^{\prime}\right\}_{i}\right. & \left.-\omega_{i}^{\prime 2}\left\{\psi^{\prime}\right\}_{j}^{\mathrm{T}}\left[m_{e}\right]\left\{\psi^{\prime}\right\}_{i}\right) \alpha_{e} \\
& =\left\{\psi^{\prime}\right\}_{j}^{\mathrm{T}}[m]\left\{\psi^{\prime}\right\}_{i} \omega_{i}^{\prime 2}-\left\{\psi^{\prime}\right\}_{j}^{\mathrm{T}}[k]\left\{\psi^{\prime}\right\}_{i}
\end{aligned}
$$

for $i, j=1,2, \ldots, n$. Equation (27) is the counterpart of eqn (8). In the redesign process the designer is also allowed to place redesign constraints on natural frequencies and normal modes. These constraints along with eqn (27) provide a system of simultaneous nonlinear implicit equations and/or inequalities in $\alpha_{e}, e=1,2, \ldots, p$. These are very complex and must also be modified like their static counterparts using the algorithms developed in Section 3. 
In general, in redesign, both static and modal dynamic constraints are imposed on the objective design. Solution algorithms used in the general redesign problem are presented in Sections 3 and 4 . If only static constraints are imposed the problem will be reduced to a static redesign problem which has been solved in Ref. 16. If only modal dynamic constraints are imposed the general redesign problem will be reduced to a dynamic redesign problem, which has been solved in Refs 11 and 14. The algorithm developed in this work, besides being able to simultaneously handle both static and modal dynamic constraints, is more accurate, faster and efficient. This is particularly the case in the manipulation of static redesign constraints which are modified as explained in the next section using a modal expansion.

\section{ALGORITHM TO MODIFY REDESIGN CONSTRAINTS}

Redesign constraints imposed on $\{\Delta u\},\left[\omega^{\prime 2}\right]$ and $\left\{\psi^{\prime}\right\}_{j}(j=1,2, \ldots, n)$ may be in the following equality or inequality form

$$
\begin{array}{cl}
u_{i}^{\prime}=u_{i}+\Delta u_{i} \leqq b_{u_{i}} & i=1,2, \ldots, s_{u} \\
\omega_{i}^{\prime 2}=\omega_{i}^{2}+\Delta \omega_{i}^{2} \leqq b_{\omega_{i}} & i=1,2, \ldots, s_{\omega}
\end{array}
$$

\section{INCREMENT $l$}

PROBLEM DEFINITION: $\quad$ Eqns: $\quad$ (88), (89), (90), (91), (92)

Unknowns: $\quad \alpha_{e_{1}}, e=1,2, \ldots, p$

Constraints: $S=S_{\omega}+S_{\phi}+2 p$

INPUT: $\quad b_{\omega_{i}}^{(l)}, i=1,2, \ldots, S_{\omega}$

$$
\begin{aligned}
& b_{\phi_{k i}}^{(l)},(k, i) \in J\left(1,2, \ldots, S_{\phi}\right) \\
& {\left[k_{e}\right]_{l},\left[m_{e}\right]_{l},[\phi]_{l},\left[-\omega_{i-}^{2}\right]_{l}}
\end{aligned}
$$

SOLVER: Solve for $\alpha_{e}, e=1,2, \ldots, p$ (Section 4)

PREDICTION: Compute $[c]$ from eqn (62)

Compute $[\delta \phi]_{l}$ from eqn (59)

OUTPUT: $\quad$ Compute $\left[\phi^{\prime}\right]_{l}=[\phi]_{l}+[\delta \phi]_{l}$

Fig. 2. Predictor phase problem. 


$$
\phi_{k i}^{\prime}=\phi_{k i}+\Delta \phi_{k i} \lesseqgtr b_{\phi_{k i}} \quad(k, i) \in J\left(1,2, \ldots, S_{\phi}\right)
$$

where $b_{u_{i}}, b_{\omega_{i}}$ and $b_{\phi_{k i}}$ are quantities specified by the designer and $S_{u}, S_{\omega}$ and $S_{\phi}$ are the number of constraints imposed on elements of $\{\Delta u\}$, $\left[-\Delta \omega^{2}-\right]$ and $[\Delta \phi]$, respectively. In all cases the expressions provided for $\Delta u_{i}$ by eqn (8) and for $\omega_{i}^{\prime 2}$ and $\phi_{k i}^{\prime}$ by eqn (27) must be modified before being used in the redesign process. These expressions are in a nonlinear implicit form which can be altered efficiently by the incremental predictor-corrector scheme explained below and diagrammatically shown in Figs 2 and 3.

In the predictor and corrector phases certain linear approximations are used. This implies that imposed constraints on structural response as defined by eqns (28), (29) and (30), as well as expected fractional changes $\alpha_{e} s$, are small. If they are small they can be applied in one incremental step. If they are not small they must be applied in $N$ small increments as follows. Let $u_{i,}^{\prime}, \omega_{i,}^{\prime 2}, \phi_{k i,}^{\prime}$ be redesign goals (desirable values) of $i$ th component of displacement vector, $i$ th eigenvalue, and $k$ th component of $i$ th eigenvector, respectively, in increment $l$, with $l=1,2, \ldots N$. Then we have

$$
\begin{array}{rrrl}
u_{i_{i}}^{\prime} & i=1,2, \ldots, S_{u} & u_{i_{N}}^{\prime}=u_{i}^{\prime} \\
\omega_{i i_{i}}^{\prime 2} & i=1,2, \ldots, S_{\omega} & \omega_{i N}^{\prime 2}=\omega_{i}^{\prime 2} \\
\phi_{k_{i i}}^{\prime} & (k, i) \in J\left(1,2, \ldots, S_{\phi}\right) & \phi_{k_{i_{N}}}^{\prime}=\phi_{k i}^{\prime}
\end{array}
$$

\section{INCREMENT $l$}

PROBLEM DEFINITION: Eqns: $\quad$ (93), (94), (95), (96), (97)

Unknowns: $\quad \alpha_{e}, e=1,2, \ldots, p$

Constraints: $S=S_{u}+n_{\mathrm{T}} S_{\omega}+2 p$

INPUT: $\quad b_{u_{i}}^{(l)}, i=1,2, \ldots, S_{u}$

$b_{\omega_{i}}^{(l)}, i=1,2, \ldots, S_{\omega}$

$\left[k_{e}\right]_{l},\left[m_{e}\right]_{l},\left[-\omega_{i}^{2}\right]_{l},\left[\phi^{\prime}\right]_{l}$ from predictor phase

SOLVER: Solve for $\alpha_{e l}, e=1,2, \ldots, p$ (Section 4)

CORRECTION: Compute $\left[k^{\prime}\right]_{l}$ from eqns (3) and (7)

Compute $\left[m^{\prime}\right]_{l}$ from eqns (21) and (24)

Compute $\left[-\omega_{i}^{\prime 2}\right]_{l}$ using Rayleigh's quotient

Compute $\left\{u^{\prime}\right\}_{l}$ from eqn (80)

Fig. 3. Corrector phase problem. 
Further we assume that the above intermediate values form geometric sequences defined by eqns (34)-(36):

$$
\begin{aligned}
u_{i_{l}}^{\prime} & =u_{i_{i_{-1}}}^{\prime}\left(1+r_{u}\right) \\
\omega_{i_{l}}^{\prime 2} & =\omega_{i_{i_{-1}}}^{\prime 2}\left(1+r_{\omega}\right) \\
\phi_{k i_{l}}^{\prime} & =\phi_{k_{i_{l-1}}}^{\prime}\left(1+r_{\phi}\right)
\end{aligned}
$$

where $u_{i_{0}}^{\prime}=u_{i}, \omega_{i_{0}}^{\prime 2}=\omega_{i}^{2}$ and $\phi_{k i_{0}}^{\prime}=\phi_{k i}$.

Combining eqns (31) and (34), (32) and (35), (33) and (36) we get respectively eqns (37), (38) and (39):

$$
\begin{aligned}
& \left(1+r_{u}\right)^{N}=\frac{u_{i}^{\prime}}{u_{i}} \\
& \left(1+r_{\omega}\right)^{N}=\frac{\omega_{i}^{\prime 2}}{\omega_{i}^{2}} \\
& \left(1+r_{\phi}\right)^{N}=\frac{\phi_{k i}^{\prime}}{\phi_{k i}}
\end{aligned}
$$

To ascertain small errors in the linear approximation used in the predictor phase, $\left|r_{u}\right|,\left|r_{\omega}\right|$ and $\left|r_{\phi}\right|$ are set $\leqslant r$, where in practice $r=0.07$. This inequality along with eqns (37), (38) and (39) yields the following three lower bounds for $N$ :

$$
\begin{gathered}
N \geqslant \frac{\ln \left(u_{i}^{\prime} / u_{i}\right)}{\ln (1+r)}=\frac{\ln \left(b_{u_{i}} / u_{i}\right)}{\ln (1+r)} \\
N \geqslant \frac{\ln \left(\omega_{i}^{\prime 2} / \omega_{i}^{2}\right)}{\ln (1+r)}=\frac{\ln \left(b_{\omega_{i}} / \omega_{i}^{2}\right)}{\ln (1+r)} \\
N \geqslant \frac{\ln \left(\phi_{k i}^{\prime} / \phi_{k i}\right)}{\ln (1+r)}=\frac{\ln \left(b_{\phi_{k i}} / \phi_{k i}\right)}{\ln (1+r)}
\end{gathered}
$$

$N$ is the smallest integer which satisfies these three inequalities. Then $r_{u}$, $r_{\omega}$ and $r_{\phi}$ can be defined using eqns (37), (38) and (39), respectively.

The following symbols are also defined. At each increment $l$ the values achieved by the redesign algorithm are, in general, not equal to the goals, $u_{i,}^{\prime}, \omega_{i}^{\prime 2}, \phi_{k i t}^{\prime}$. Instead, values which are used as baseline values for the next increment $(l+1)$ are achieved. These are denoted by $u_{i_{l+1}}, \omega_{i_{i+1}}^{2}$ and $\phi_{k i l+1}$. For the first increment we have $u_{i_{1}}=u_{i}, \omega_{i_{1}}^{2}=\omega_{i}^{2}$ and $\phi_{k i_{1}}=\phi_{k i}$.

Let $\delta$ denote difference between values of a quantity in any two sequential steps in the incremental algorithm. Then, the following differences can be defined: 


$$
\begin{gathered}
\{\delta u\}_{l}=\left\{u^{\prime}\right\}_{l}-\{u\}_{l} \\
{\left[-\delta \omega^{2}-\right]_{l}=\left[-\omega^{\prime 2}-\right]_{l}-\left[-\omega^{2}-\right]_{l}} \\
{[\delta \phi]_{l}=\left[\phi^{\prime}\right]_{l}-[\phi]_{l}}
\end{gathered}
$$

which implies that the modal vectors satisfy the following relation:

$$
\begin{aligned}
\{\delta \psi\}_{j_{l}} & =\left\{\psi^{\prime}\right\}_{j_{l}}-\{\psi\}_{j_{l}} \\
{[\delta k]_{l} } & =\left[k^{\prime}\right]_{l}-[k]_{l} \\
{[\delta m]_{l} } & =\left[m^{\prime}\right]_{l}-[m]_{l}
\end{aligned}
$$

Further, the counterparts of the bounds provided by eqns (28), (29) and (30) must be defined for each increment $l$. That is, $b_{u_{i}}^{(l)}, b_{\omega_{i}}^{(l)}$ and $b_{\phi_{k i}}^{(l)}$ must be defined in the following equality or inequality forms of the redesign constraints at increment $l$ :

$$
\begin{aligned}
& u_{i_{l}}^{\prime}=u_{i_{i}}+\delta_{u_{i l}} \leqq b_{u_{i}}^{(l)} \quad i=1,2, \ldots, S_{u} \\
& \omega_{i_{i}}^{\prime 2}=\omega_{i_{i}}^{2}+\delta \omega_{i_{i}}^{2} \lesseqgtr b_{\omega_{i}}^{(l)} \quad i=1,2, \ldots, S_{\omega} \\
& \phi_{k i_{i}}^{\prime}=\phi_{k i_{i}}+\delta \phi_{k i_{i}} \leqq b_{\phi_{k i}}^{(l)} \quad(k, i) \in J\left(1,2, \ldots, S_{\phi}\right)
\end{aligned}
$$

These bounds can be defined using equations similar to (37), (38) and (39). Thus

$$
\begin{array}{ccrl}
b_{u_{i}}^{(l)}=u_{i}\left(1+r_{u}\right)^{l} & i & =1,2, \ldots, S_{u} \\
b_{\omega_{i}}^{(l)}=\omega_{i}^{2}\left(1+r_{\omega}\right)^{l} & i & =1,2, \ldots, S_{\omega} \\
b_{\phi_{k i}}^{(l)}=\phi_{k i}\left(1+r_{\phi}\right)^{l} & (k, i) & \in J\left(1,2, \ldots, S_{\phi}\right)
\end{array}
$$

Finally, bounds should be imposed on the allowable structural changes $\alpha_{e}$ s. Two kinds of bounds are used. First, for practical purposes

$$
\alpha_{e}^{-} \leqslant \alpha_{e} \leqslant \alpha_{e}^{+} \quad e=1,2, \ldots, p
$$

where $\alpha_{e}^{-}$and $\alpha_{e}^{+}$are specified lower and upper bounds for $\alpha_{e}$ and obviously $a_{e}^{-}>-1$. Second, at each increment $l$ in order to ascertain validity of the linear approximations used in both predictor and corrector phases the following bounds are imposed:

$$
-0 \cdot 1 \leqslant \alpha_{e_{l}} \leqslant 0 \cdot 1 \quad \text { for } l=1,2, \ldots, N \text { and } e=1,2, \ldots, p
$$

The bounds in (55) are directly applied to the allowable structural changes at each increment. Those in (54), however, are applied to the global $\alpha_{e} s$ and must be modified to reveal bounds applicable to the incremental $\alpha_{e}$ s. Let 


$$
1+\alpha_{e}=\prod_{l=1}^{N}\left(1+\alpha_{e_{l}}\right)
$$

Then (54) and (56) yield

$$
1+\alpha_{e}^{-} \leqslant \prod_{l=1}^{N}\left(1+\alpha_{e_{l}}\right) \leqslant 1+\alpha_{e}^{+} \quad e=1,2, \ldots, p
$$

This suggests use of the following bounds on the incremental $\alpha_{e} s$ :

$$
\frac{1+\alpha_{e}^{-}}{\prod_{q=1}^{l-1}\left(1+\alpha_{e_{q}}\right)} \leqslant 1+\alpha_{e_{l}} \leqslant \frac{1+\alpha_{e}^{+}}{\prod_{q=1}^{l-1}\left(1+\alpha_{e_{q}}\right)}
$$

where $\prod_{q=1}^{0}\left(1+\alpha_{e_{q}}\right)=1$. Of the two upper and two lower bounds imposed on $\alpha_{e_{t}}$ by (55) and (58) the dominant (stricter) ones are used.

In the following description of predictor and corrector phases, in each increment $l$, index l is omitted in order to simplify notation. It should also be emphasized that operators $\Delta$ and $\delta$ have identical functions, the only difference being that the former indicates global and possibly large change while the latter indicates incremental small change. Thus all equations involving $\Delta$ (e.g. eqns (1) and (4)) are valid for $\delta$ as well.

\section{Predictor phase}

In the predictor phase eqn (27) is linearized following the method originally proposed by Stetson ${ }^{1-4}$ and later extended by Sandström. ${ }^{5,6}$ According to this method changes in eigenvectors are expressed as

$$
[\delta \phi]=[\phi][c]^{\mathrm{T}}
$$

where $[c]$ is the matrix of admixture coefficients. It is assumed that $c_{i i}=0$ and that the rest of the coefficients are small. Numerically it is more convenient to work in the space spanned by the eigenvectors $\{\psi\}_{i}$, $i=1,2, \ldots, n$ transformed by $[c]$ rather than the eigenvectors themselves.

Applying eqns (59), (22) and (23) for changes to eigenvectors, mass matrix and eigenvalues to eqn (26) and neglecting terms of the order of $\delta^{2}$, $\delta^{3}$ and $\delta^{4}$ we get the first order perturbation equations as

$$
\{\psi\}_{j}^{\mathrm{T}}[\delta k]\{\psi\}_{i}-\{\psi\}_{j}^{\mathrm{T}}[\delta m]\{\psi\}_{i} \omega_{i}^{2} \begin{cases}=M_{i} \delta \omega_{i}^{2} & \text { for } i=j \\ =M_{j} c_{i j}\left(\omega_{i}^{2}-\omega_{j}^{2}\right) & \text { for } i \neq j\end{cases}
$$


These equations are derived assuming that the neglected terms are of higher order. This is correct only for small changes which is the case in each increment. For $i=j$ eqn (60) gives the following expression for changes to eigenvalues:

$$
\delta \omega_{i}^{2}=\frac{1}{M_{i}}\left(\{\psi\}_{i}^{\mathrm{T}}[\delta k]\{\psi\}_{i}-\{\psi\}_{i}^{\mathrm{T}}[\delta m]\{\psi\}_{i} \omega_{i}^{2}\right)
$$

For $i \neq j$ eqn (60) gives the admixture coefficients $c_{i j}$ :

$$
c_{i j}=\frac{1}{M_{j}\left(\omega_{i}^{2}-\omega_{j}^{2}\right)}\left(\{\psi\}_{j}^{\mathrm{T}}[\delta k]\{\psi\}_{i}-\omega_{i}^{2}\{\psi\}_{j}^{\mathrm{T}}[\delta m]\{\psi\}_{i}\right)
$$

Combining eqns (59) and (60) we can express the change to the $k$ th degree of freedom of the $i$ th mode as

$$
\delta \phi_{k i}=\sum_{j=1, j \neq i}^{n}\left[\frac{\phi_{k j}}{M_{j}\left(\omega_{i}^{2}-\omega_{j}^{2}\right)}\left(\{\psi\}_{j}^{\mathrm{T}}[\delta k]\{\psi\}_{i}-\omega_{i}^{2}\{\psi\}_{j}^{\mathrm{T}}[\delta m]\{\psi\}_{i}\right)\right]
$$

Introducing eqns (7) and (24) in (61) and (63) the following relations for $\delta \omega_{i}^{2}$ and $\delta \phi_{k i}$ are derived in terms of the required structural changes $\alpha_{e}$, $e=1,2, \ldots, p$ :

$$
\begin{gathered}
\delta \omega_{i}^{2}=\frac{1}{M_{i}}\left[\sum_{e=1}^{p}\left(\{\psi\}_{i}^{\mathrm{T}}\left[k_{e}\right]\{\psi\}_{i}-\omega_{i}^{2}\{\psi\}_{i}^{\mathrm{T}}\left[m_{e}\right]\{\psi\}_{i}\right) \alpha_{e}\right] \\
\delta \phi_{k i}=\sum_{e=1}^{p}\left[\sum_{j=1 . j \neq i}^{n} \frac{\phi_{k j}}{M_{j}\left(\omega_{i}^{2}-\omega_{j}^{2}\right)}\left(\{\psi\}_{j}^{\mathrm{T}}\left[k_{e}\right]\{\psi\}_{i}-\omega_{i}^{2}\{\psi\}_{j}^{\mathrm{T}}\left[m_{e}\right]\{\psi\}_{i}\right)\right] \alpha_{e}
\end{gathered}
$$

where $n_{\mathrm{r}}$ is the number of modes of the baseline structure used in redesign. Obviously $n_{\mathrm{r}}$ has to be high enough to achieve an accurate approximation of $\delta \phi_{k i}$. The predictor phase algorithm is summarized in Fig. 2.

In the predictor phase only dynamic redesign constraints are used to compute a first order approximation to the structural changes $\alpha_{e}$ s. These constraints may be in the form of equalities or inequalities as indicated in eqns (28)-(30). Depending on the number $p$ of unknown $\alpha_{e} s$ and the number of equality and/or inequality constraints $\left(S_{\omega}+S_{\phi}\right)$, several different solution algorithms may be used as explained in Section 4.

There is one subcase in the above algorithm which requires special attention - specifically the case of repeated eigenvalues. If, say, $\omega_{i}=\omega_{j}$, $c_{i j}$ cannot be defined by equation (62). This problem of repeated eigenvalues is not new. It instigates similar complications in sensitivitybased optimal design methods. ${ }^{19-21}$ In this work the problem of repeated 
eigenvalues is resolved as follows. It is assumed that the designer would like his objective structure to possess similar properties and therefore preserve any eigenvalue repetition. Then it is expected that change to mode $j$ will not depend on mode $i$ just as it does not depend on mode $j$ itself. In the definition of the admixture coefficient matrix by equation (59) it was assumed that $c_{i i}=0$. Similarly we can impose the restriction on $[c]$ that $c_{i j}=0$ for $\omega_{i}=\omega_{j}$.Other elements in [c] may also be zero. These can be computed by RESTRUCT as in the offshore tower application in Section 6. Coefficients that are zero or nearly zero are purged from the system of nonlinear constraints by RESTRUCT to increase the speed and accuracy of solution.

\section{Corrector phase}

In the corrector phase a first order approximation to the objective eigenvectors is computed using eqns (65) and (23). These approximate eigenvectors of the objective structure are subsequently used in eqn (27) to provide better approximation to the elemental changes $\alpha_{e}$ s. Specifically we get $\left(n_{\mathrm{r}} \times S_{\omega}\right)$ number of redesign constraints of the following form:

$$
\begin{aligned}
& \sum_{e=1}^{p}\left(\left\{\psi^{\prime}\right\}_{j}^{\mathrm{T}}\left[k_{e}\right]\left\{\psi^{\prime}\right\}_{i}-\omega_{i}^{\prime 2}\left\{\psi^{\prime}\right\}_{j}^{\mathrm{T}}\left[m_{e}\right]\left\{\psi^{\prime}\right\}_{i}\right) \alpha_{e} \\
& =\left\{\psi^{\prime}\right\}_{j}^{\mathrm{T}}[m]\left\{\psi^{\prime}\right\}_{i} \omega_{i}^{\prime 2}-\left\{\psi^{\prime}\right\}_{j}^{\mathrm{T}}[k]\left\{\psi^{\prime}\right\}_{i} \quad j=1,2, \ldots, n_{\mathrm{r}}
\end{aligned}
$$

or

$$
\begin{aligned}
\delta \omega_{i}^{2}= & -\omega_{i}^{2}+\frac{1}{\left\{\psi^{\prime}\right\}_{j}^{\mathrm{T}}[m]\left\{\psi^{\prime}\right\}_{i}} \times \\
& {\left[\left\{\sum_{e=1}^{p}\left(\left\{\psi^{\prime}\right\}_{j}^{\mathrm{T}}\left[k_{e}\right]\left\{\psi^{\prime}\right\}_{i}-\omega_{i}^{\prime 2}\left\{\psi^{\prime}\right\}_{j}^{\mathrm{T}}\left[m_{e}\right]\left\{\psi^{\prime}\right\}_{i} \alpha_{e}\right\}\right.\right.} \\
& \left.+\left\{\psi^{\prime}\right\}_{j}^{\mathrm{T}}[k]\left\{\psi^{\prime}\right\}_{i}\right] \quad j=1,2, \ldots, n_{\mathrm{r}}
\end{aligned}
$$

At this point the static redesign constraints must be introduced and taken into account in computing the $\alpha_{e}$ values. For this purpose eqn (8) is modified using the following approach.

Let $\left\{Q^{\prime}\right\}$ be defined by eqn (67):

$$
\left\{u^{\prime}\right\}=\left[\phi^{\prime}\right]\left\{Q^{\prime}\right\}
$$

where $\left\{Q^{\prime}\right\}$ is the transformed displacement vector. Application of eqn (67) to (2) gives 


$$
\left[k^{\prime}\right]\left[\phi^{\prime}\right]\left\{Q^{\prime}\right\}=\{f\}
$$

Premultiplying eqn (68) by $\left[\phi^{\prime}\right]^{\mathrm{T}}$ gives

$$
\left[\phi^{\prime}\right]^{\mathrm{T}}\left[k^{\prime}\right]\left[\phi^{\prime}\right]\left\{Q^{\prime}\right\}=\left[\phi^{\prime}\right]^{\mathrm{T}}\{f\}
$$

Since $\left[\phi^{\prime}\right]^{\mathrm{T}}\left[k^{\prime}\right]\left[\phi^{\prime}\right]=\left[K^{\prime}-\right]$, eqn (69) becomes

$$
\begin{gathered}
{\left[K^{\prime}-\right]\left\{Q^{\prime}\right\}=\left[\phi^{\prime}\right]^{\mathrm{T}}\{f\}} \\
\left\{Q^{\prime}\right\}=\left[K^{\prime}\right]^{-1}\left[\phi^{\prime}\right]^{\mathrm{T}}\{f\}
\end{gathered}
$$

Since $\left[K^{\prime},\right]^{-1}=\left[-1 / K_{j j}^{\prime}\right]$, eqn (71) becomes

$$
\left\{Q^{\prime}\right\}=\left[-1 / K_{j j}^{\prime}\right]\left[\phi^{\prime}\right]^{\mathrm{T}}\{f\}
$$

Combination of eqns (72) and (67) yields

$$
\begin{aligned}
& \left\{u^{\prime}\right\}=\left[\phi^{\prime}\right] \quad\left[-1 / K_{j j-}^{\prime}\right]\left[\phi^{\prime}\right]^{\mathrm{T}} \quad\{f\} \\
& n \times 1 \quad n \times n_{r} n_{r} \times n_{r} \quad n_{r} \times n n \times 1
\end{aligned}
$$

Thus the components of vector $\left\{u^{\prime}\right\}$ can be expressed as

$$
u_{i}^{\prime}=\sum_{m=1}^{n_{\mathrm{f}}}\left[\frac{\phi_{i m}^{\prime}}{K_{m}^{\prime}} \sum_{j=1}^{n} \phi_{j m}^{\prime} f_{j}\right]
$$

To calculate $K_{m}^{\prime}$, eqns (3), (7) and (19) are combined to produce

$$
\begin{aligned}
{\left[-K^{\prime}-\right] } & =\left[\phi^{\prime}\right]^{\mathrm{T}}\left([k]+\sum_{e=1}^{p}\left[k_{e}\right] \alpha_{e}\right)\left[\phi^{\prime}\right] \\
& =\left[\phi^{\prime}\right]^{\mathrm{T}}[k]\left[\phi^{\prime}\right]+\sum_{e=1}^{p} \alpha_{e}\left[\phi^{\prime}\right]^{\mathrm{T}}\left[k_{e}\right]\left[\phi^{\prime}\right]
\end{aligned}
$$

or

$$
K_{m}^{\prime}=\left\{\psi^{\prime}\right\}_{m}^{\mathrm{T}}[k]\left\{\psi^{\prime}\right\}_{m}+\sum_{e=1}^{p} \alpha_{e}\left\{\psi^{\prime}\right\}_{m}^{\mathrm{T}}\left[k_{e}\right]\left\{\psi^{\prime}\right\}_{m} \quad m=1,2, \ldots n_{\mathrm{r}}
$$

Combination of eqns (76) and (74) yields

$$
u_{i}^{\prime}=\sum_{m=1}^{n_{\mathrm{T}}}\left[\frac{\phi_{i m}^{\prime}}{\left.\left\{\psi^{\prime}\right\}_{m}^{\mathrm{T}}[k]\left\{\psi^{\prime}\right\}_{m}+\sum_{e=1}^{p} \alpha_{e}\left\{\psi^{\prime}\right\}_{m}^{\mathrm{T}}\left[k_{e}\right] \psi^{\prime}\right\}_{m}}\left(\sum_{j=1}^{n} \phi_{j m}^{\prime} f_{j}\right)\right]
$$

Equation (77) shows that the displacement vector of the objective system can be expressed in terms of eigenvectors of the objective system, 
the stiffness matrix of baseline structure, the fractional changes of stiffness properties and the applied force vector. Let

$A_{m}=\sum_{j=1}^{n} \phi_{j m}^{\prime} f_{j}, B_{m}=\left\{\psi^{\prime}\right\}_{m}^{\mathrm{T}}[k]\left\{\psi^{\prime}\right\}_{m}$ and $C_{m e}=\left\{\psi^{\prime}\right\}_{m}^{\mathrm{T}}\left[k_{e}\right]\left\{\psi^{\prime}\right\}_{m}$

Then eqn (77) becomes

$$
u_{i}^{\prime}=\sum_{m=1}^{n_{\mathrm{I}}} \frac{\phi_{i m}^{\prime} A_{m}}{B_{m}+\sum_{e=1}^{p} C_{m e} \alpha_{e}}=\sum_{m=1}^{n_{\mathrm{r}}} \frac{1}{1+\frac{1}{B_{m}} \sum_{e=1}^{p} C_{m e} \alpha_{e}} \frac{\phi^{\prime}{ }_{i m} A_{m}}{B_{m}}
$$

If at each step we assume small changes from the previous step, $B_{m}$ will be much greater than $\sum_{e=1}^{p} C_{m e} \alpha_{e}$. Therefore we can linearize eqn (79) as follows:

$$
\begin{aligned}
& u_{i}^{\prime} \approx \sum_{m=1}^{n_{\mathrm{T}}}\left[\frac{\phi_{i m}^{\prime} A_{m}}{B_{m}}\left(1-\frac{1}{B_{m}} \sum_{e=1}^{p} C_{m e} \alpha_{e}\right)\right] \\
& =\sum_{m=1}^{n_{\mathrm{F}}} \frac{\phi^{\prime}{ }_{i m} A_{m}}{B_{m}}-\sum_{m=1}^{n_{\mathrm{F}}}\left[\frac{\phi_{i m}^{\prime} A_{m}}{B_{m}^{2}}\left(\sum_{e=1}^{p} C_{m e} \alpha_{e}\right)\right]
\end{aligned}
$$

or

$$
\Delta u_{i}=-u_{i}+\sum_{m=1}^{n_{\mathrm{T}}} \frac{\phi_{i m}^{\prime} A_{m}}{B_{m}}-\sum_{e=1}^{p}\left(\sum_{m=1}^{n_{\mathrm{T}}} \frac{\phi_{i m}^{\prime} A_{m}}{B_{m}^{2}} C_{m e}\right) \alpha_{e}
$$

In the corrector phase of the redesign process the static redesign equality or inequality constraints are introduced. Let $S_{u}$ be the total number of static constraints. Depending on the number $p$ of unknown $\alpha_{e}$ s and the total number of static and/or dynamic equality and/or inequality redesign constraints $\left(n_{\mathrm{r}} \times S_{\omega}+S_{u}\right)$ several different solution algorithms may be used as explained in Section 4.

At the end of each increment $\left\{u^{\prime}\right\}$ and $\left[\omega^{\prime 2}\right]$ are computed using eqns (80) and (66). These values are used along with the values of $\left[\phi^{\prime}\right]-$ computed in the predictor phase - at the beginning of the next increment as baseline values. The corrector phase algorithm is summarized in Fig. 3. 


\section{COMPUTATION OF STRUCTURAL CHANGES}

The problem of redesign by perturbation as defined in Section 2 consists of constraint equations (28), (29) and (30), the dynamic perturbation equation (27) and the static perturbation equation (8). That is, the problem of redesign by perturbation is reduced to problem P1.

P1: Redesign by perturbation

$$
\begin{gathered}
u_{i}^{\prime}=u_{i}+\Delta u_{i} \leqq b_{u_{i}} \quad i=1,2, \ldots, S_{u} \\
\omega_{i}^{\prime 2}=\omega_{i}^{2}+\Delta \omega_{i}^{2} \lesseqgtr b_{\omega_{i}} \quad i=1,2, \ldots, S_{\omega} \\
\phi_{k i}^{\prime}=\phi_{k i}+\Delta \phi_{k i} \lesseqgtr b_{\phi_{k i}} \quad(k, i) \in J\left(1,2, \ldots, S_{\phi}\right) \\
\sum_{e=1}^{p}\left(\left\{\psi^{\prime}\right\}_{j}^{\mathrm{T}}\left[k_{e}\right]\left\{\psi^{\prime}\right\}_{i}-\omega_{i}^{\prime 2}\left\{\psi^{\prime}\right\}_{j}^{\mathrm{T}}\left[m_{e}\right]\left\{\psi^{\prime}\right\}_{i}\right) \alpha_{e} \\
=\left\{\psi^{\prime}\right\}_{j}^{\mathrm{T}}[m]\left\{\psi^{\prime}\right\}_{i} \omega_{i}^{\prime 2}-\left\{\psi^{\prime}\right\}_{j}^{\mathrm{T}}[k]\left\{\psi^{\prime}\right\}_{i} \quad i, j=1,2, \ldots, n \\
\{\delta u\}=-\sum_{e=1}^{p}\left([k]+\sum_{e=1}^{p}\left[k_{e}\right] \alpha_{e}\right)^{-1}\left[k_{e}\right]\{u\} \alpha_{e} \\
\alpha_{e}^{-} \leqslant \alpha_{e} \leqslant \alpha_{e}^{+} \quad e=1,2, \ldots, p
\end{gathered}
$$

In problem $\mathrm{P} 1$ the unknowns are $\alpha_{e}, e=1,2, \ldots, p$.

As explained in Section 2 this problem is very complicated because of the nonlinear implicit form of expressions (85) and (86). The incremental predictor-corrector scheme developed in Section 3 is used to apply $b_{u_{i}}, b_{\omega_{i}}$ and $b_{\phi_{k i}}$ incrementally and simplify expressions (85) and (86). At each increment $l(l=1,2, \ldots, N)$ two different problems arise; problem P2 appears in the predictor phase and problem $\mathrm{P} 3$ in the corrector phase. Both are defined below.

P2: Predictor phase problem (increment $l, l=1,2, \ldots, N$ )

$$
\begin{aligned}
& \omega_{i_{i}}^{\prime 2}=\omega_{i,}^{2}+\delta \omega_{i,}^{2} \lesseqgtr b_{\omega_{i}}^{(l)} \quad i=1,2, \ldots, S_{\omega} \\
& \phi_{k i_{i}}^{\prime}=\phi_{k i_{i}}+\delta \phi_{k i /} \leqq b_{\phi_{k i}}^{(l)} \quad(k, i) \in J\left(1,2, \ldots, S_{\phi}\right)
\end{aligned}
$$


where

$$
\delta \omega_{i_{i}}^{2}=\left(\frac{1}{M_{i}}\left[\sum_{e=1}^{p}\left(\{\psi\}_{i}^{\mathrm{T}}\left[k_{e}\right]\{\psi\}_{i}-\omega_{i}^{2}\{\psi\}_{i}^{\mathrm{T}}\left[m_{e}\right]\{\psi\}_{i}\right) a_{e}\right]\right)_{l}
$$

and

$$
\begin{gathered}
\delta \phi_{k i_{i}}=\left(\sum _ { e = 1 } ^ { p } \left[\sum _ { j = 1 , j \neq i } ^ { n _ { r } } \frac { \phi _ { k j } } { M _ { j } ( \omega _ { i } ^ { 2 } - \omega _ { j } ^ { 2 } ) } \left(\{\psi\}_{j}^{\mathrm{T}}\left[k_{e}\right]\{\psi\}_{i}\right.\right.\right. \\
\left.\left.\left.-\omega_{i}^{2}\{\psi\}_{j}^{\mathrm{T}}\left[m_{e}\right]\{\psi\}_{i}\right)\right] \alpha_{e}\right)_{l} \\
\max \left\{(1-0 \cdot 1), \frac{1+\alpha_{e}^{-}}{\prod_{q=1}^{1-1}\left(1+\alpha_{e_{q}}\right)}\right\} \leqslant 1+\alpha_{e_{i}} \leqslant \min \left\{(1+0 \cdot 1), \frac{1+\alpha_{e}^{+}}{\prod_{q=1}^{1-1}\left(1+\alpha_{e_{q}}\right)}\right\} \\
e=1,2, \ldots, p
\end{gathered}
$$

In problem P2 the unknowns are $\alpha_{e}, e=1,2, \ldots, p$, and the total number of constraints is $S=S_{\omega}+S_{\phi}+2 p$. This problem and its solution algorithm are presented in Fig. 2.

P3: Corrector phase problem (increment $l, l=1,2, \ldots, N$ )

$$
\begin{aligned}
u_{i_{i}}^{\prime}=u_{i_{l}}+\delta u_{i_{i}} \leqq b_{u_{i}}^{(l)} & i=1,2, \ldots, S_{u} \\
\omega_{i_{l}}^{\prime 2}=\omega_{i l}^{2}+\delta \omega_{i_{i}}^{2} \leqq b_{\omega_{i}}^{(l)} & i=1,2, \ldots, S_{\omega}
\end{aligned}
$$

where

$$
\begin{aligned}
\delta \omega_{i_{i}}^{2} & =-\omega_{i_{i}}^{2}+\left(\frac{1}{\left\{\psi^{\prime}\right\}_{j}^{\mathrm{T}}[m]\left\{\psi^{\prime}\right\}_{i}} \times\right. \\
& {\left[\sum_{e=1}^{p}\left\{\left\{\psi^{\prime}\right\}_{j}^{\mathrm{T}}\left[k_{e}\right]\left\{\psi^{\prime}\right\}_{i}-\omega_{i}^{\prime 2}\left\{\psi^{\prime}\right\}_{j}^{\mathrm{T}}\left[m_{e}\right]\left\{\psi^{\prime}\right\}_{i} \alpha_{e}\right\}\right.} \\
& \left.\left.+\left\{\psi^{\prime}\right\}_{j}^{\mathrm{T}}[k]\left\{\psi^{\prime}\right\}_{i}\right]\right)_{l} \quad j=1,2, \ldots, n_{\mathrm{r}}
\end{aligned}
$$

and

$$
\delta u_{i_{i}}+u_{i_{1}}=u_{i_{i}}^{\prime}=\left(\sum_{m=1}^{n_{\mathrm{r}}} \frac{\phi_{i m}^{\prime} A_{m}}{B_{m}}-\sum_{e=1}^{p}\left(\sum_{m=1}^{n_{\mathrm{r}}} \frac{\phi_{i m}^{\prime} A_{m}}{B_{m}^{2}} C_{m e}\right) \alpha_{e}\right),
$$




$$
\begin{gathered}
\max \left\{(1-0 \cdot 1), \frac{1+\alpha_{e}^{-}}{\prod_{q=1}^{1-1}\left(1+\alpha_{e_{q}}\right)}\right\} \leqslant 1+\alpha_{e_{l}} \leqslant \min \left\{(1+0 \cdot 1), \frac{1+\alpha_{e}^{+}}{\prod_{q=1}^{l-1}\left(1+\alpha_{e_{q}}\right)}\right\} \\
e=1,2, \ldots, p
\end{gathered}
$$

In problem P3 the unknowns are again $\alpha_{e}, e=1,2, \ldots, p$, and the total number of constraints is $S=S_{u}+n_{r} S_{\omega}+2 p$. This problem and its solution algorithm are summarized in Fig. 3.

Problems P2 and P3 are solved at each increment. Their form is similar and the number of unknowns is in both equal to $p$. They differ only in the number of equality and inequality constraints. Due to their similarity problems P2 and P3 can be solved for the unknown $\alpha_{e_{l}}$ s using the same solvers which are described below.

Let $S_{\mathrm{e}}$ and $S_{\mathrm{i}}$ be the number of equality and inequality constraints, respectively. Further let $S_{\mathrm{b}}$ be the number of upper and lower bound inequality constraints imposed on $\alpha_{e_{l}} s$. Depending on the relation between $S_{\mathrm{e}}, S_{\mathrm{i}}, S_{\mathrm{b}}$ and $p$ the following problems are identified.

(i) $\quad p>S_{\mathrm{e}}$ : In this case an optimization problem with $p$ design variables, $S_{\mathrm{e}}$ equality constraints and $\left(S_{\mathrm{i}}+S_{\mathrm{b}}\right)$ inequality constraints must be solved.

(ii $a) \quad p=S_{\mathrm{e}}$ and $S_{\mathrm{i}}=S_{\mathrm{b}}=0$ : In this case a system of simultaneous linear equations must be solved.

(ii $\beta) \quad p=S_{\mathrm{c}}$ and $\left(S_{\mathrm{i}}+S_{\mathrm{b}}\right)>0$ : In this case the problem is in general overconstrained. To solve it, first the linear solver of case (ii $\alpha$ ) is used and then the $\left(S_{\mathrm{i}}+S_{\mathrm{b}}\right)$ inequality constraints are checked. If they are satisfied the solution will be accepted; if not the problem will be solved approximately by the minimum error algorithm in (iii).

(iii) $p<S_{\mathrm{e}}$ : In this case the minimum error algorithm is used to approximately satisfy all equality and inequality constraints.

The solvers used in the above problems are briefly described below.

\section{Optimization solvers}

In case (i) above the problem in general has infinite solutions and an optimization criterion must be used to select one solution. Then the problem becomes an optimization problem with $p$ unknowns, $S_{\mathrm{e}}$ linear equality constraints, $S_{\mathrm{i}}$ linear inequality constraints and $S_{\mathrm{b}}=2 p$ simple bound constraints. Depending on the type of criterion used, one of the following three solvers may be employed: 
(i) The Simplex linear programming algorithm $\mathrm{m}^{22}$ for a linear criterion.

(ii) A quadratic programming algorithm $\mathrm{m}^{23,24}$ for a quadratic criterion like the following minimum change criterion:

$$
\min \left(\sum_{e=1}^{p} \alpha_{e_{l}}^{2}\right)
$$

which is most often used in applications. ${ }^{11.12 .14 .16}$

(iii) A conjugate gradient nonlinear algorith ${ }^{25}$ combined with an external penalty technique ${ }^{26}$ for a nonlinear criterion of order higher than quadratic. In this case a nonlinear programming method based on sequential quadratic programming is also used. ${ }^{27}$

The purpose of using so many different optimization algorithms at the expense of a significantly larger code is the following. The constraint equations in their present form are linear. The objective is in general quadratic but may be linear or nonlinear of order higher than quadratic. Algorithms that take advantage of specific properties of the problem in hand are dedicated algorithms and consequently faster and more accurate. We have found after many applications that it is preferable to expand RESTRUCT to include the Simplex algorithm and a quadratic algorithm based on Simplex, rather than solving all problems using the more general - and therefore not dedicated - nonlinear algorithms mentioned in (iii) above.

\section{Linear equation solver}

In case (ii $a$ ) explained above the problem is reduced to a set of simultaneous linear equations which are solved by the classical Cholesky factorization method.

Minimum error solver

This case arises in (ii $\beta$ ) and (iii) above. The algorithm works as follows. ${ }^{28}$ The $S_{\mathrm{i}}$ inequality constraints are considered as equalities and the $S_{\mathrm{b}}$ bound constraints are ignored. The resulting problem has $\left(S_{\mathrm{e}}+S_{\mathrm{i}}\right)$ equalities and $p$ unknowns and is represented by eqn (99):

$$
\begin{aligned}
& {[A] \quad\{x\}=\{b\}} \\
& S \times p p \times 1 \quad S \times 1
\end{aligned}
$$

Since $S>p$ eqn (99) has no solution. Premultiplying by $[A]^{\mathrm{T}}$ and solving for $\{x\}$ we get

$$
\{x\}=\left([A]^{\mathrm{T}}[A]\right)^{-1}[A]^{\mathrm{T}}\{b\}
$$


This approximate solution $\{x\}$ minimizes the norm

$$
|\{b\}-[A]\{x\}|
$$

\section{COMPUTER IMPLEMENTATION}

Most problems in structural analysis and design of marine structures are large-scale problems that can be solved only by general or special purpose finite element codes. For a redesign tool of such structures to be

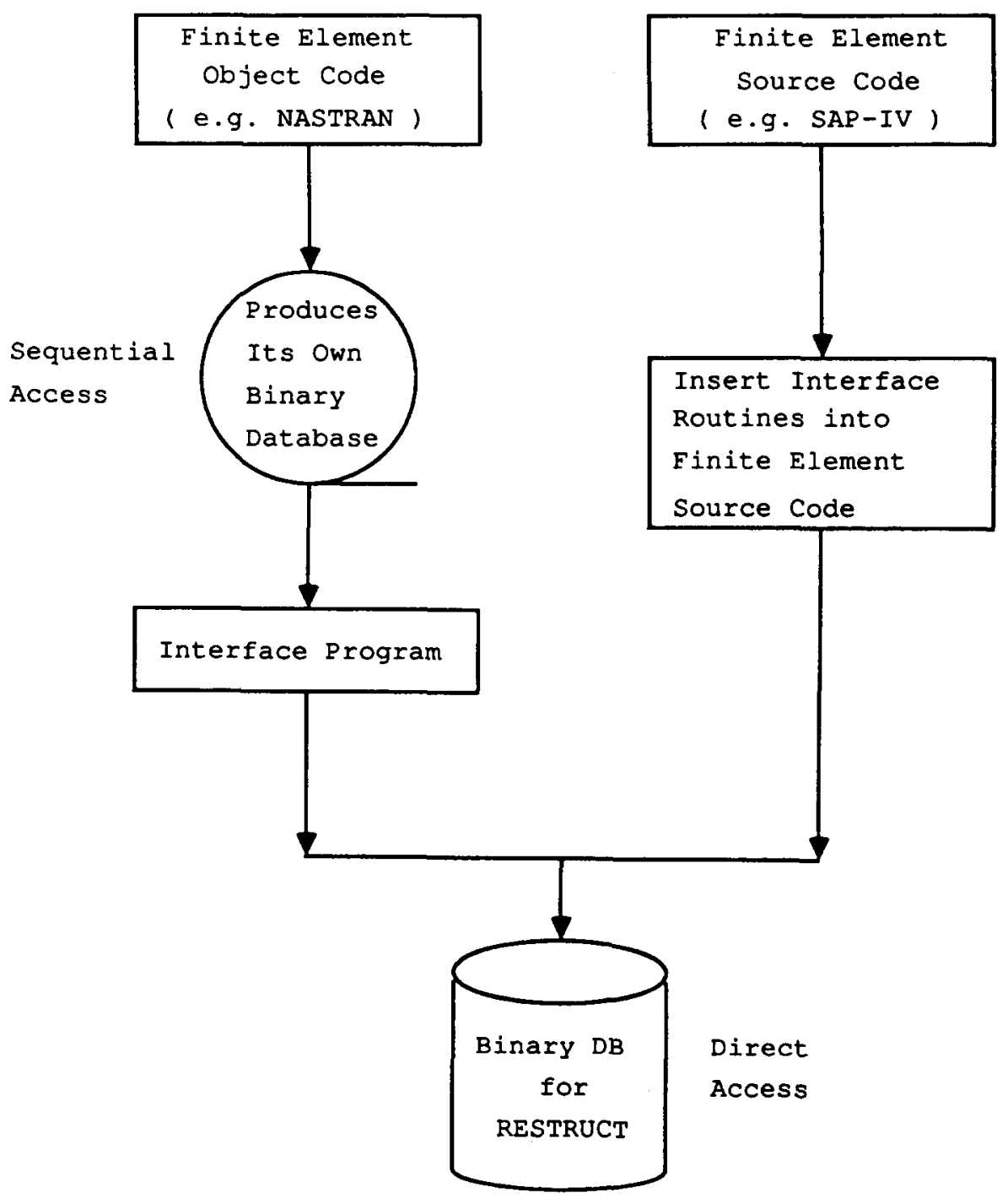

Fig. 4. Block diagram for production of database. 


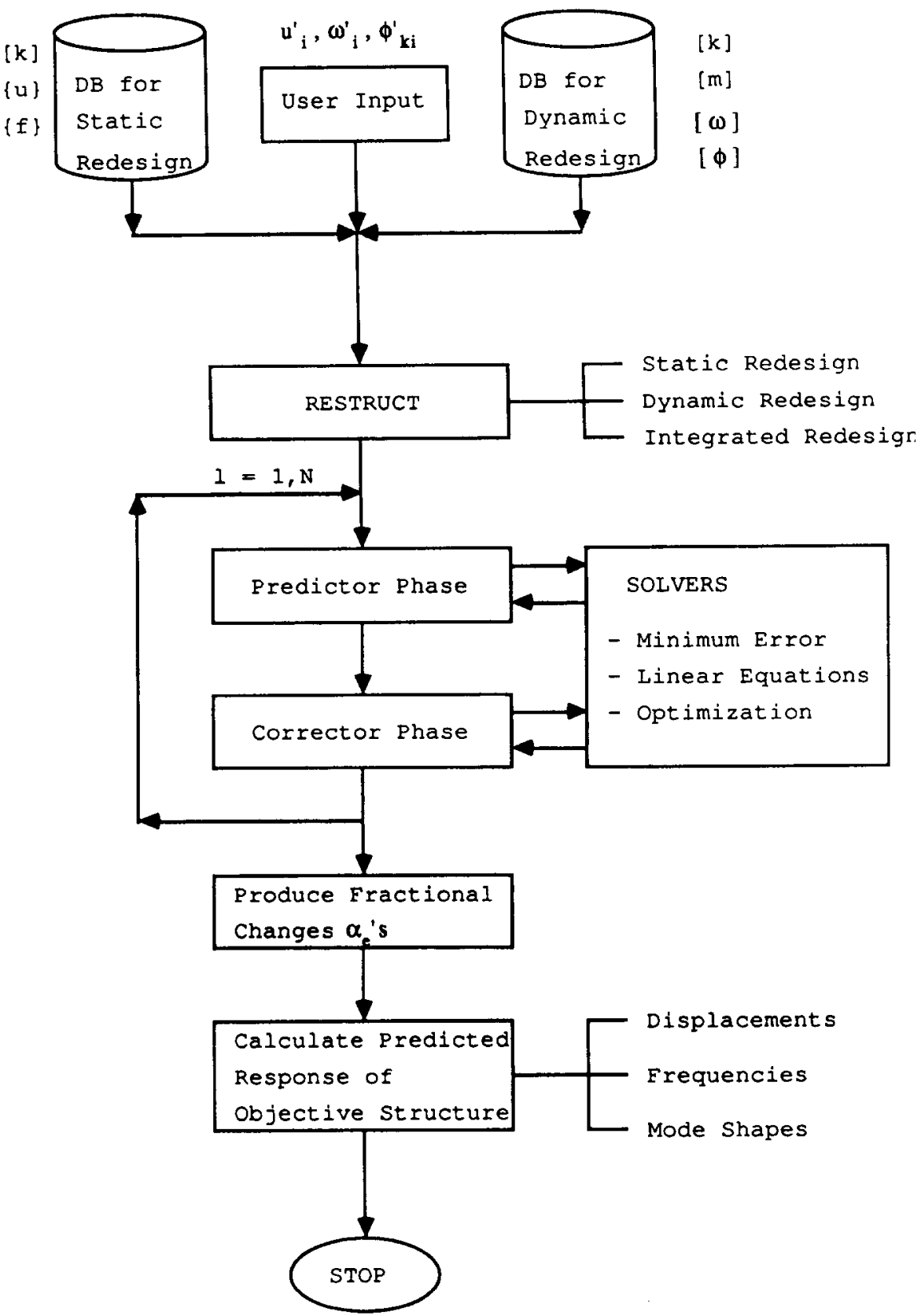

Fig. 5. Block diagram for redesign procedure (RESTRUCT). 
of practical use it must serve as a postprocessor to such codes. Computer code RESTRUCT (about 19500 Fortran commands) works as a postprocessor to general or special purpose FE programs. Thus it provides a high accuracy, fully automated structural redesign tool which can be used for solution of practical problems.

Some key ideas for computer implementation are diagrammatically summarized in Figs 1, 4 and 5. Figure 1 shows the relation between structural analysis and redesign. Any FE code may be used to compute the structural response of a given structure and fill in the redesign database. If response is not satisfactory, redesign must be performed using the database produced in the baseline analysis step.

Figure 4 shows the way the database for redesign is produced. If a FE code produces its own type of database the latter will be changed to produce the RESTRUCT database through an interface program. Such is the case when NASTRAN is used for the baseline structure. NASTRAN produces a database in binary code which can be read using a dedicated language (DMAP) and transformed to the RESTRUCT database which is also in binary code. This process is necessary because the source code of NASTRAN is not available. If the source code of the general or special purpose FE program, which is used for the static and/ or dynamic analysis of the baseline structure is available, interface routines will be appended to its source code to produce the RESTRUCT database.

Figure 5 shows the redesign procedure implemented in RESTRUCT. It has three options, one for static, one for dynamic and one for static/ dynamic (integrated) redesign. The latter uses data stored in the RESTRUCT database by both static and dynamic analyses runs. Following reading of the database and the user supplied input, RESTRUCT constructs static and/or dynamic constraint equations using the algorithm developed in Section 3. The resulting constraint problem is then formulated and solved for the required structural changes according to the methods explained in Section 4. Finally, RESTRUCT uses the produced structural changes to compute displacements, frequencies and eigenmodes of the objective structure.

\section{REDESIGN APPLICATIONS}

Several numerical applications are used in this section to test the applicability and efficiency of RESTRUCT as well as some practical aspects related to conflicting redesign requirements, number of modes 
used in modal expansions, repeated eigenvalues and size of the FE structural model. Two structures are used, a simple 5-element clampedhinged beam and a 192-d.o.f. offshore tower.

\section{Beam problem}

The simple 5-element clamped-hinged beam shown in Fig. 6 is first used to illustrate some of the basic features of RESTRUCT and the developed perturbation-based redesign methodology. Analysis of the baseline structure by NASTRAN produced a first frequency $F_{1}=\omega_{1} / 2 \pi=29 \cdot 14 \mathrm{~Hz}$ and a static displacement for the fourth node $d_{4}=7.09 \mathrm{~mm}$. Eight cases are analyzed for various values of the number of eigenmodes used in redesign, the objective values of $d_{4}$ and $F_{1}$, and the number $p$ of allowable structural changes. Results are shown in Tables 1-8. By comparing results in these tables the following conclusions can be drawn:

(i) Trying to satisfy both static and dynamic constraints by changing only the beam stiffness - as in Cases 2, 4, 6-does not produce good results. Results improve significantly when the cross-sectional area is also allowed to change, as in Cases 1, 3,5 . This is so because change in $A_{i}, i=1,2, \ldots, 5$, results in mass change. It is, in general, impossible to satisfy both dynamic and static constraints by changing only structural stiffness. Change to mass is also needed.

(ii) In Cases 1,2,3 and 4, $F_{1}$ and $d_{4}$ are assigned objective values that are in some sense compatible, that is $F_{1}^{\prime}>F_{1}$ and $d_{4}{ }^{\prime}<d_{4}$. Both indicate that the objective structure is stiffer than the baseline structure. Thus, results in Cases 2 and 4, where only the beam stiffness and not the mass (area) is allowed to change, are reasonable.
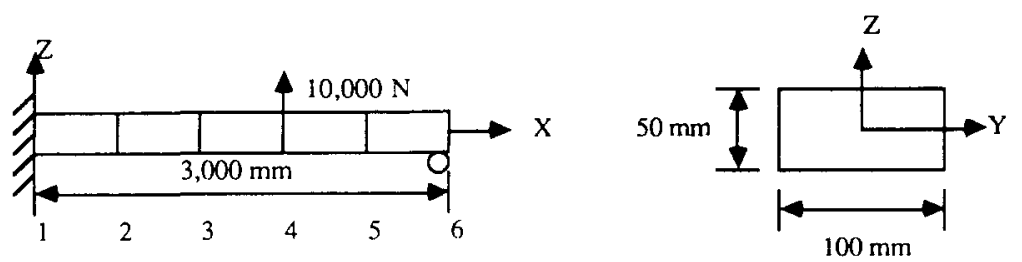

$$
\begin{array}{ll}
E=2.07 \times 10^{5} \mathrm{mPa} & v=0.3 \\
\rho=7.833 \times 10^{-9} \mathrm{~N} \mathrm{~s}^{2} / \mathrm{mm}^{4} & \\
I_{y}=1.042 \times 10^{6} \mathrm{~mm}^{4} & I_{\%}=4.170 \times 10^{6} \mathrm{~mm}^{4}
\end{array}
$$

Fig. 6. Five element clamped-hinged beam model. 


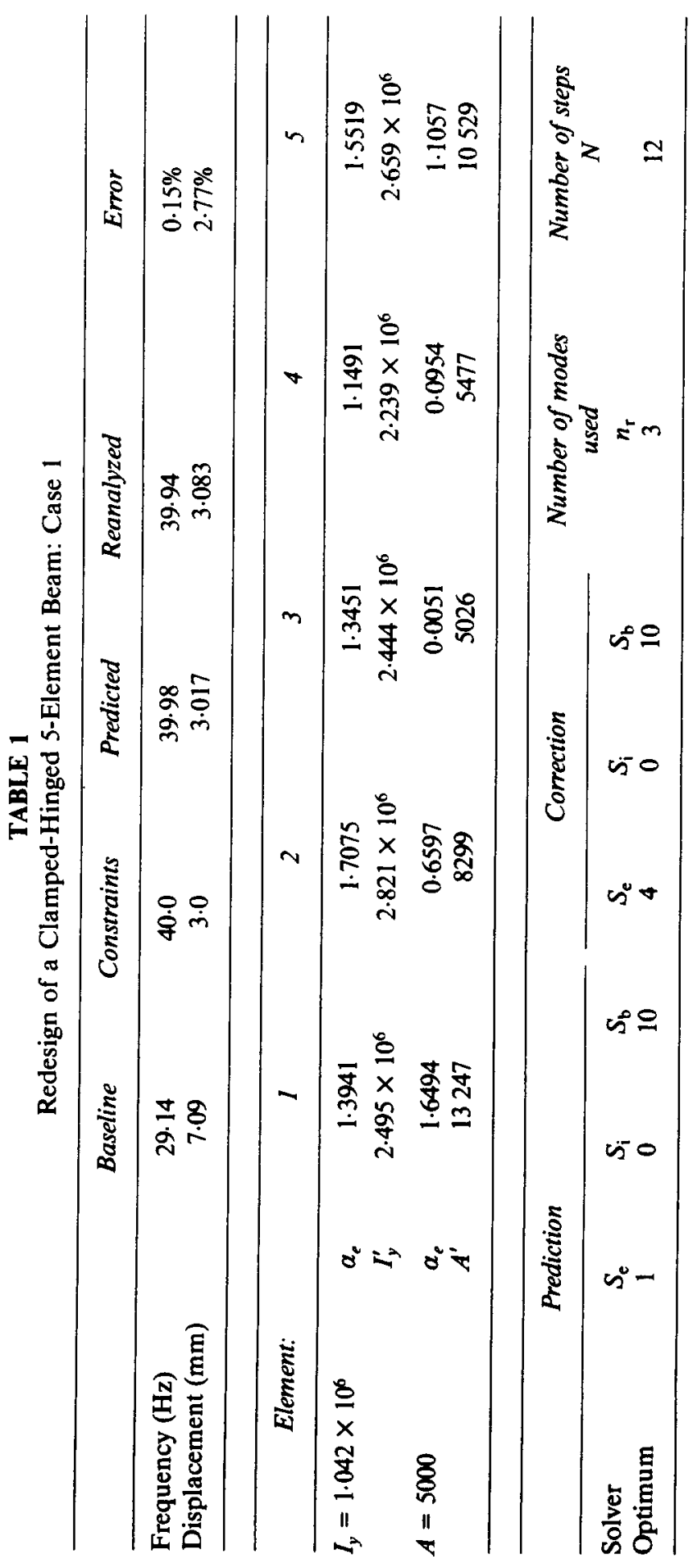




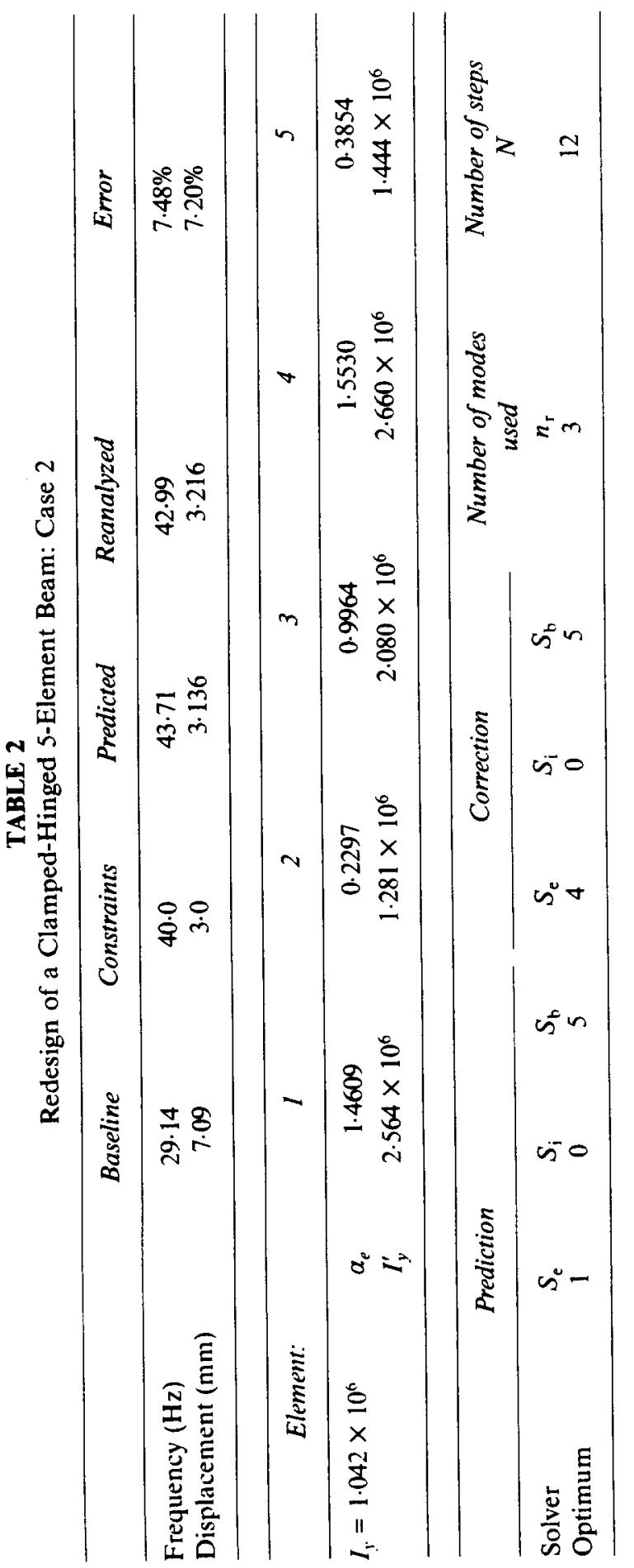




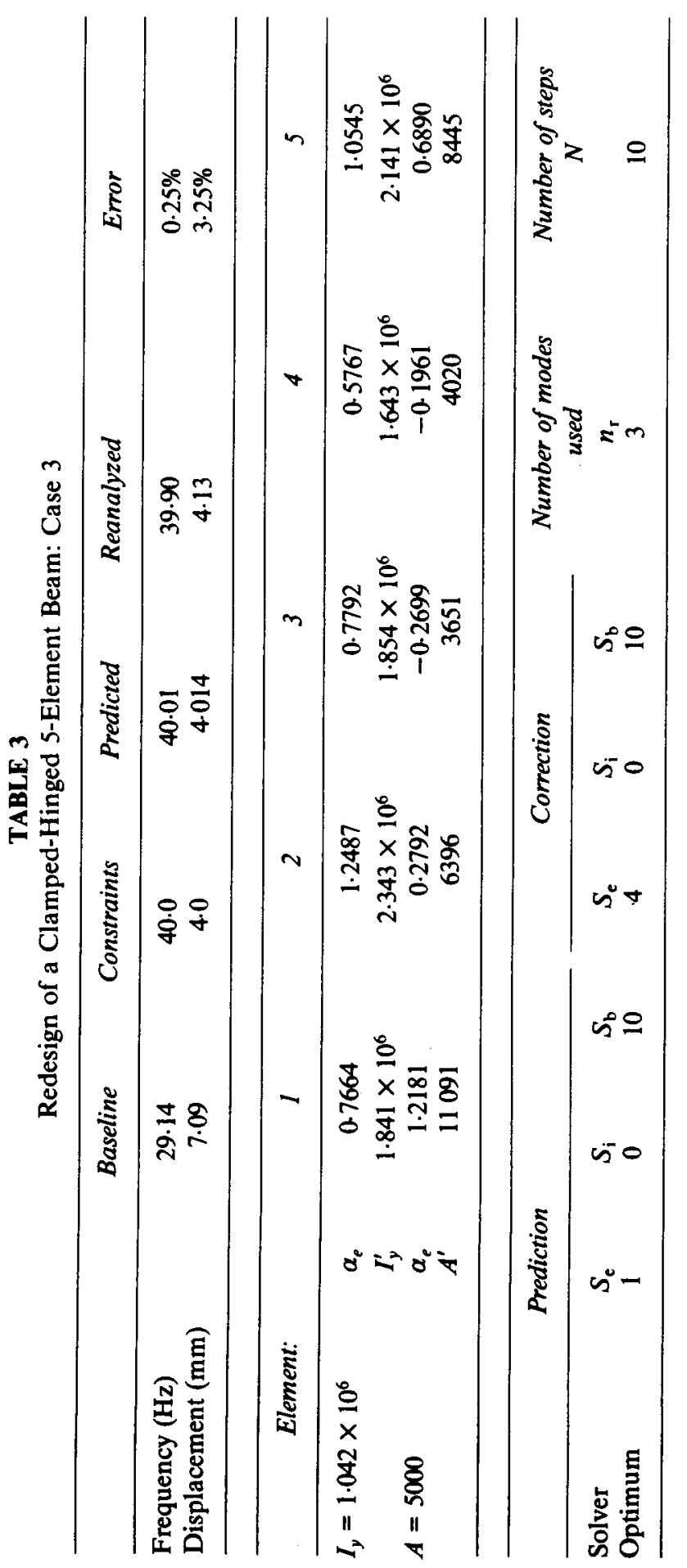




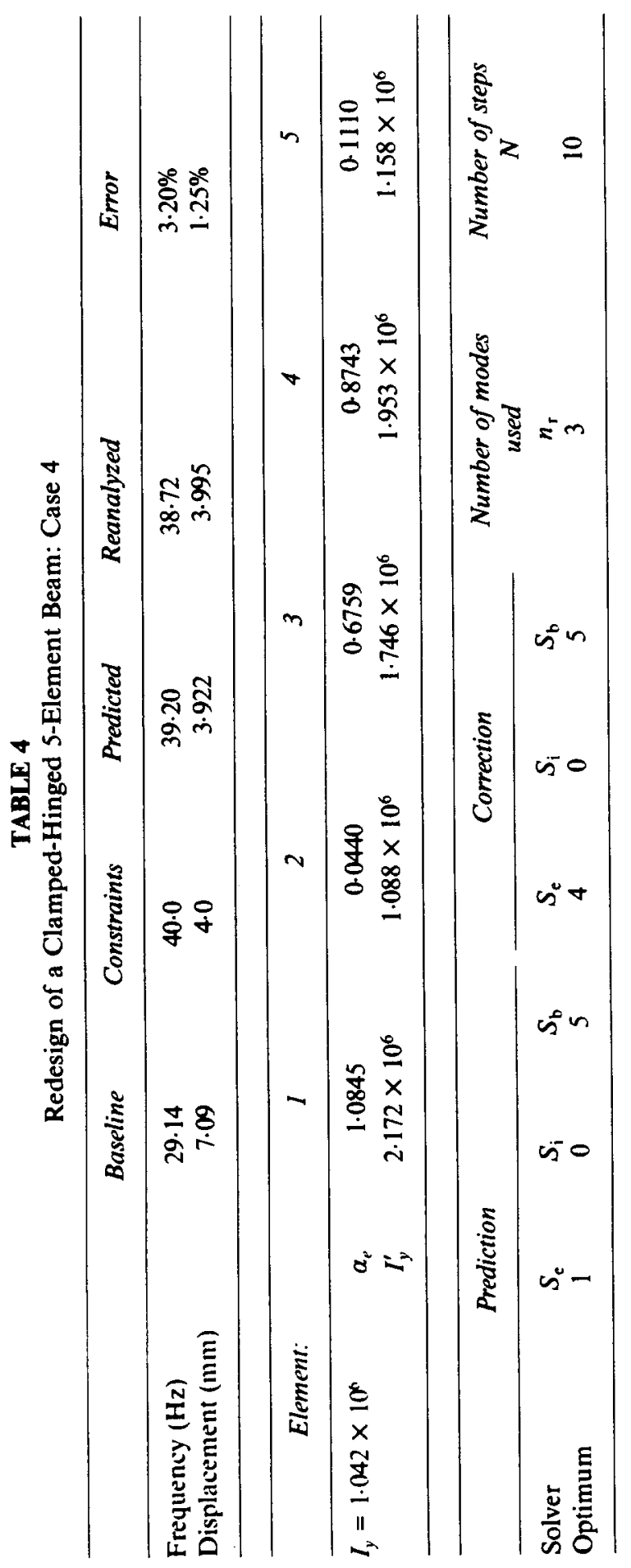


Redesign of marine structures by perturbation

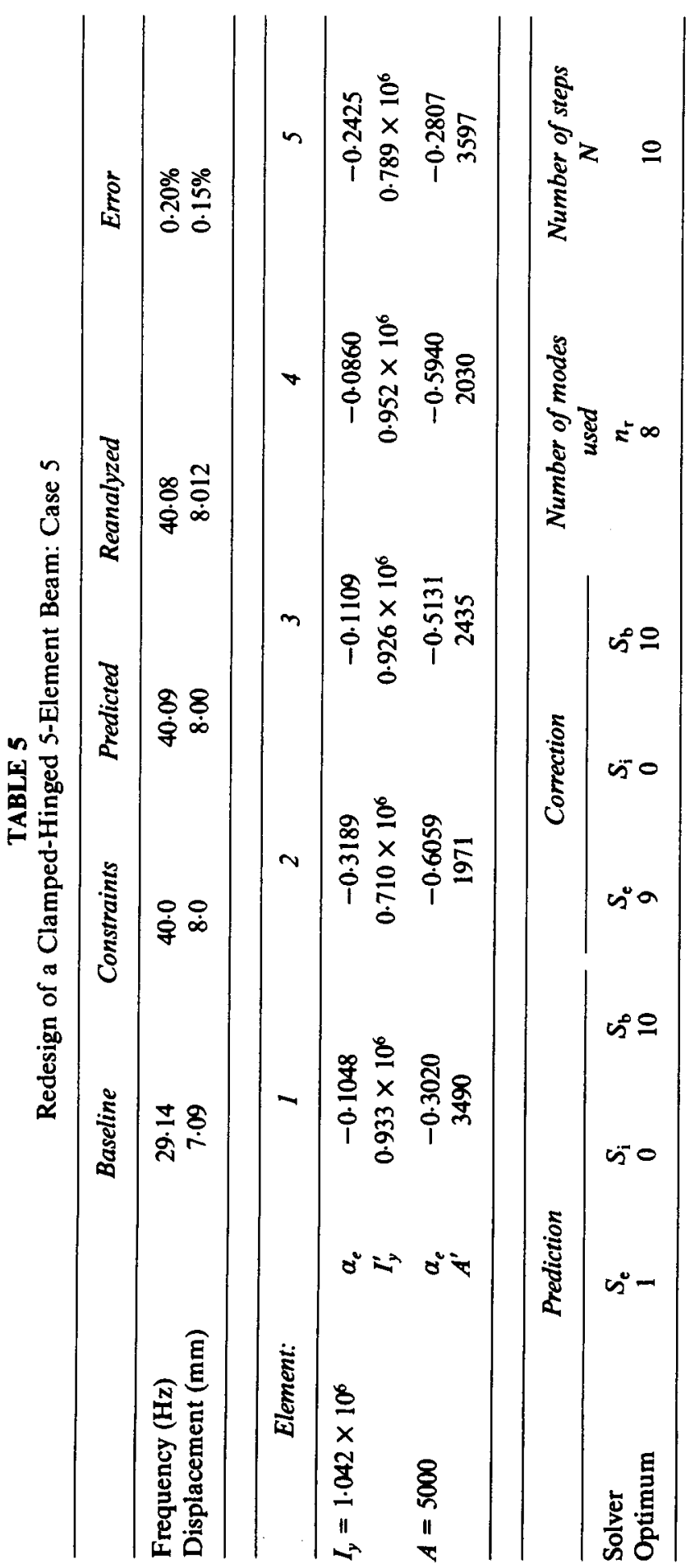




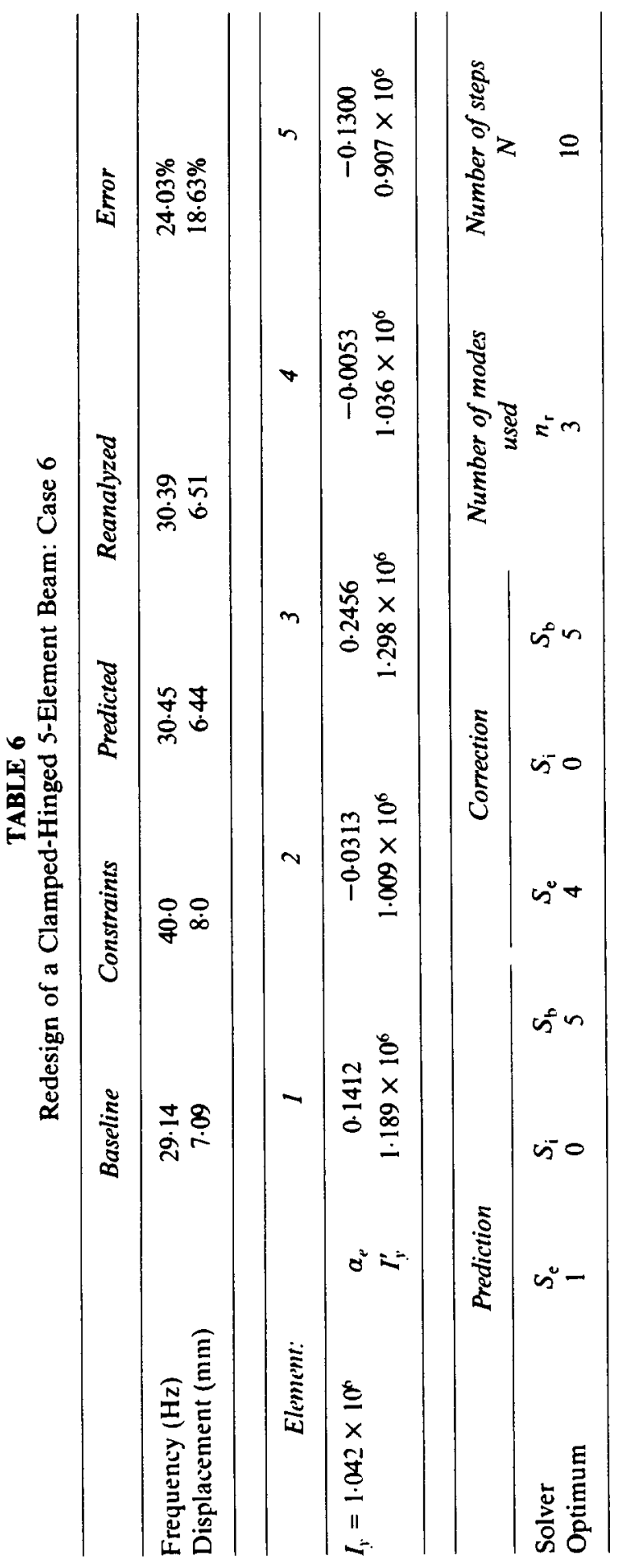


Redesign of marine structures by perturbation

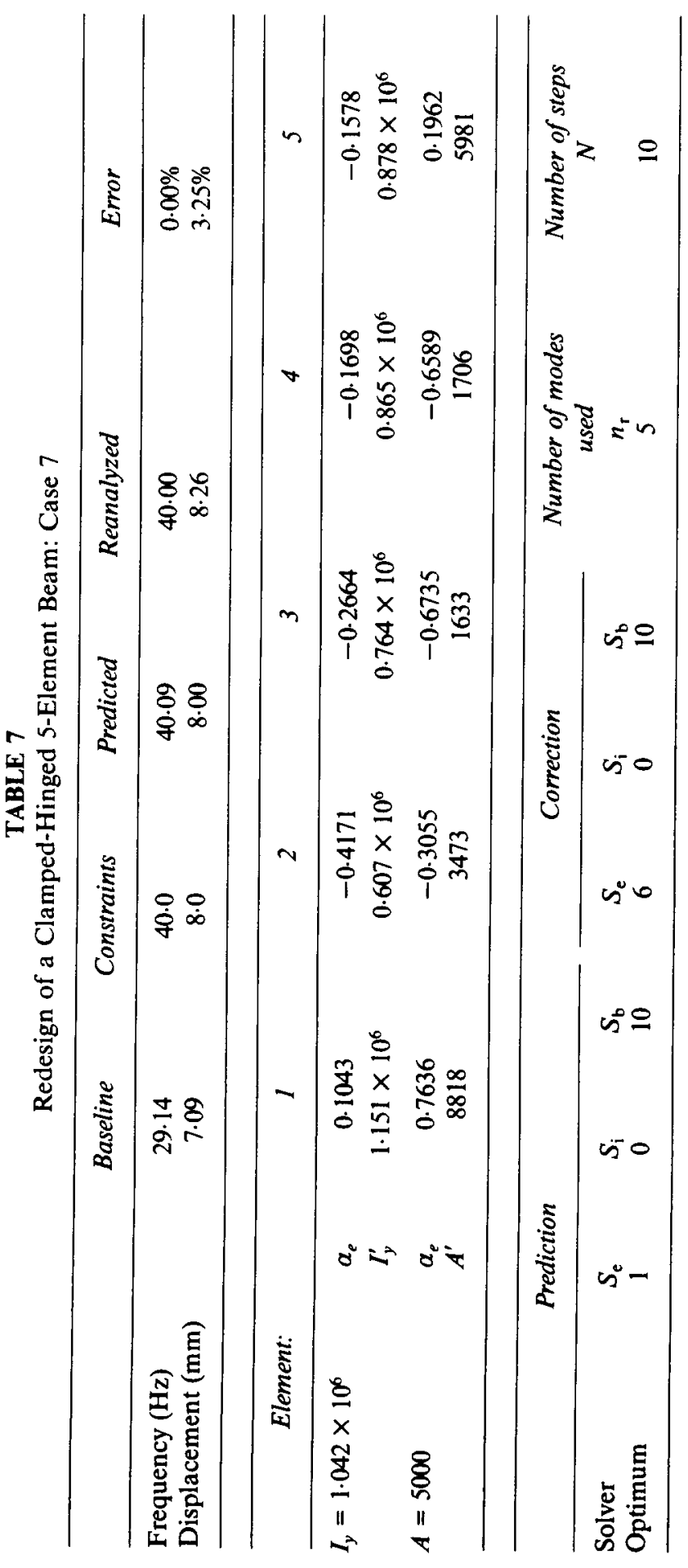




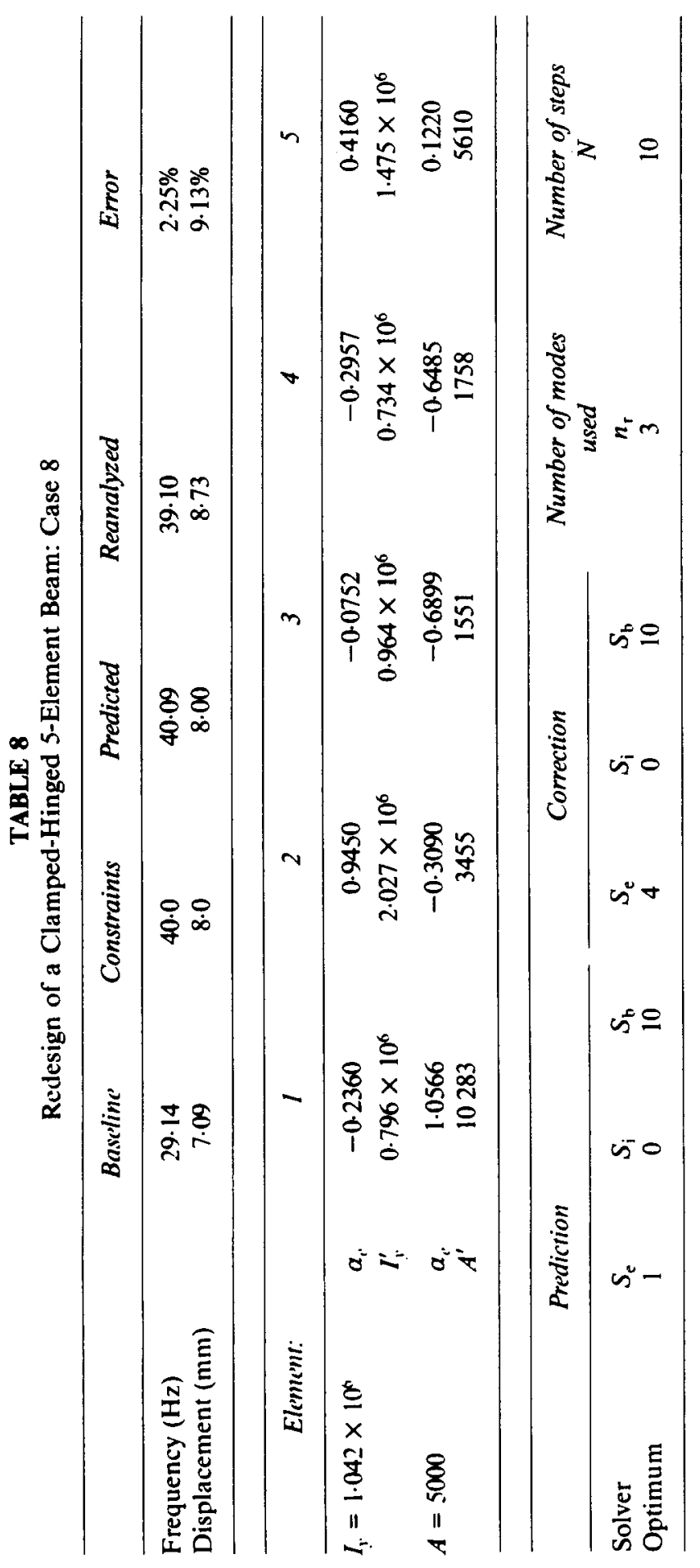


(iii) On the contrary in Case 6 where $F_{1}^{\prime}>F_{1}$ and $d_{4}^{\prime}>d_{4}$ the first constraint requires the objective structure to be stiffer while the second less stiff. Allowing only change in stiffness shows that RESTRUCT cannot satisfy both constraints and produces a minimum error solution. These two constraints are incompatible in this case.

(iv) Comparing Cases 5, 7,8 it can be seen that the accuracy of the redesign algorithm reduces with the number of eigenmodes used in the modal series. This is expected since only three modes, as in Case 8, are not expected to contain most of the structure's modal energy.

\section{Offshore tower problem}

The 8-legged offshore tower shown in Fig. 7 is $229.5 \mathrm{ft} \mathrm{high}^{\dagger}$ and operates in $150 \mathrm{ft}$ water depth. The tower at the base is $125 \mathrm{ft}$ square and tapers linearly to $75 \mathrm{ft}$ square at the deck. The FE model of the tower is composed of 104 circular tubular beam elements and has 192 degrees of freedom. Loading on the tower is due to:

(i) 200 long ton ${ }^{\dagger}$ deck load which is applied to the structure as uniformly distributed loads at the deck nodal points.

(ii) Wave hydrodynamic forces calculated for a design wave of $600 \mathrm{ft}$ length and $20 \mathrm{ft}$ height using Morison's equation. The wave propagates in the $x$ direction.

(iii) Wind current in the $x$ direction with linear velocity profile of 2 knots at the mean free surface waterline and zero at the sea bed.

Static and dynamic analyses by NASTRAN produced (i) maximum displacement in the $x$-direction at node 11 (shown in Fig. 7) equal to 2.2739 in, ${ }^{\dagger}$ and (ii) a repeated first natural frequency $\omega_{1}=\omega_{2}=4.695 \mathrm{rad} / \mathrm{s}$ $\left(F_{1}=F_{2}=0.7472 \mathrm{~Hz}\right)$. Our redesign goals are to raise the first natural frequency above $5.3401 \mathrm{rad} / \mathrm{s}(0.85 \mathrm{~Hz})$ and reduce static displacement at node 11 . Two redesign problems are solved. In the first the displacement upperbound is set equal to 1.86 in and in the second equal to 1.71 in.

For redesign purposes the tower is subdivided into 6 substructures described in Table 9. Two variables are allowed to change in each substructure. Specifically for bending and torsional stiffness, changes to area moment of inertia are allowed; for mass and axial stiffness, changes to a rea a re allowed. Let $I, J$ and $A$ be the moment of inertia, polar moment

${ }^{\dagger} 1 \mathrm{in}=25.4 \mathrm{~mm} ; 1 \mathrm{ft}=0.3048 \mathrm{~m} ; 1$ long ton $=1.01605$ tonne. 


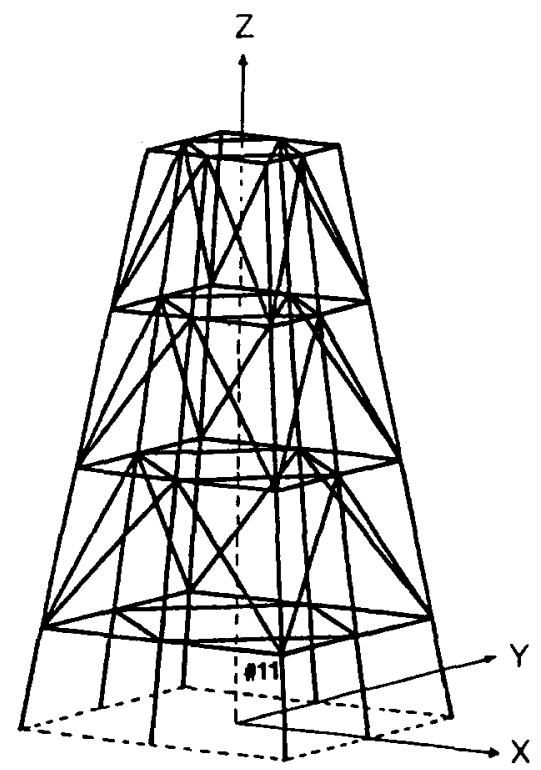

Fig. 7. Offshore tower with 8 legs and cross bracings.

of inertia and area respectively. Then we have

$$
\begin{aligned}
& I=\frac{\pi\left(D_{\mathrm{o}}^{4}-D_{\mathrm{i}}^{4}\right)}{64} \\
& J=\frac{\pi\left(D_{\mathrm{o}}^{4}-D_{\mathrm{i}}^{4}\right)}{32}=2 I \\
& A=\frac{\pi\left(D_{\mathrm{o}}^{2}-D_{\mathrm{i}}^{2}\right)}{4}
\end{aligned}
$$

where $D_{\mathrm{o}}, D_{\mathrm{i}}$ are the outer and inner tubular element diameters respectively. Let $I^{\prime}, J^{\prime}$ and $A^{\prime}$ be the corresponding quantities of the objective structure. Then $\alpha_{I}$ and $\alpha_{A}$, the allowable fractional changes to $I$ and $A$ respectively, are defined by the following equations

$$
\begin{aligned}
I^{\prime} & =I\left(1+\alpha_{I}\right) \\
J^{\prime} & =J\left(1+\alpha_{I}\right) \\
A^{\prime} & =A\left(1+\alpha_{A}\right)
\end{aligned}
$$

The total number of allowable changes is $p=12$, that is fractional changes $\alpha_{I}$ and $\alpha_{A}$ are allowed in each structural group. From $\alpha_{I}$ and $\alpha_{A}$, $D_{\mathrm{o}}^{\prime}$ and $D_{\mathrm{i}}^{\prime}$ can be computed using equations (108) and (109):

$$
D_{\mathrm{o}}^{\prime}=\sqrt{\left(a^{2}+b\right) / 2 a}
$$




$$
D_{\mathrm{i}}^{\prime}=\sqrt{\left(b-a^{2}\right) / 2 a}
$$

where

$$
\begin{aligned}
& a=\left(1+\alpha_{A}\right)\left(D_{\mathrm{o}}^{2}-D_{\mathrm{i}}^{2}\right) \\
& b=\left(1+\alpha_{l}\right)\left(D_{\mathrm{o}}^{4}-D_{\mathrm{i}}^{4}\right)
\end{aligned}
$$

The six substructures for the offshore tower, their redesign variables, and $D_{\mathrm{o}}$ and $D_{\mathrm{i}}$ for each substructure are shown in Table 9.

For the two redesign problems considered the results are summarized in Table 10 and evaluated in Table 11. The results in Table 10 show that substructures 1,4 and 5 must be altered significantly in order to achieve the designer's goals. The remaining substructures are only slightly modified in both problems. In both problems the number of design variables $p(=12)$ is greater than the number of equations in both predictor and corrector phases. The optimality criterion used to select the optimal redesign is that of minimum change between baseline and objective structures. An interesting byproduct of Redesign 1 is the reduction of the offshore tower weight, that is, by increasing the stiffness

\begin{tabular}{|c|c|c|c|c|c|}
\hline $\begin{array}{l}\text { Substructure } \\
\text { number }\end{array}$ & $\begin{array}{c}\text { Design } \\
\text { variable } \\
a_{e}\end{array}$ & $\begin{array}{l}\text { Substructure } \\
\text { description }\end{array}$ & $\begin{array}{c}D_{\mathrm{o}} \\
{\text { (in })^{a}}\end{array}$ & $\begin{array}{c}D_{i} \\
(\text { in })^{a}\end{array}$ & $\begin{array}{l}\text { Number of } \\
\text { elements }\end{array}$ \\
\hline 1 & $\begin{array}{l}a_{1}\left(\alpha_{l}\right) \\
\alpha_{2}\left(\alpha_{A}\right)\end{array}$ & $\begin{array}{l}\text { Legs below first } \\
\text { bracing }\end{array}$ & 30 & 29 & 8 \\
\hline 2 & $\begin{array}{l}\alpha_{3}\left(\alpha_{1}\right) \\
\alpha_{4}\left(\alpha_{A}\right)\end{array}$ & $\begin{array}{l}\text { Legs between first } \\
\text { and second bracing }\end{array}$ & 24 & 23 & 8 \\
\hline 3 & $\begin{array}{l}\alpha_{5}\left(\alpha_{l}\right) \\
\alpha_{6}\left(\alpha_{A}\right)\end{array}$ & $\begin{array}{l}\text { Legs above second } \\
\text { bracing }\end{array}$ & 24 & 23 & 16 \\
\hline 4 & $\begin{array}{l}\alpha_{7}\left(\alpha_{1}\right) \\
\alpha_{8}\left(\alpha_{A}\right)\end{array}$ & Horizontal bracing & 19 & 18.25 & 32 \\
\hline 5 & $\begin{array}{r}\alpha_{9}\left(\alpha_{I}\right) \\
\alpha_{10}\left(a_{A}\right)\end{array}$ & $\begin{array}{l}\text { Horizontal cross } \\
\text { bracing }\end{array}$ & 20 & 19.25 & 16 \\
\hline 6 & $\begin{array}{l}a_{11}\left(\alpha_{l}\right) \\
a_{12}\left(\alpha_{A}\right)\end{array}$ & $\begin{array}{l}\text { Vertical cross } \\
\text { bracing }\end{array}$ & 24 & $23 \cdot 25$ & 24 \\
\hline
\end{tabular}

TABLE 9

Offshore Tower Substructures, Redesign Variables, and Dimensions

${ }^{a_{1}}$ in $=25.4 \mathrm{~mm}$. 


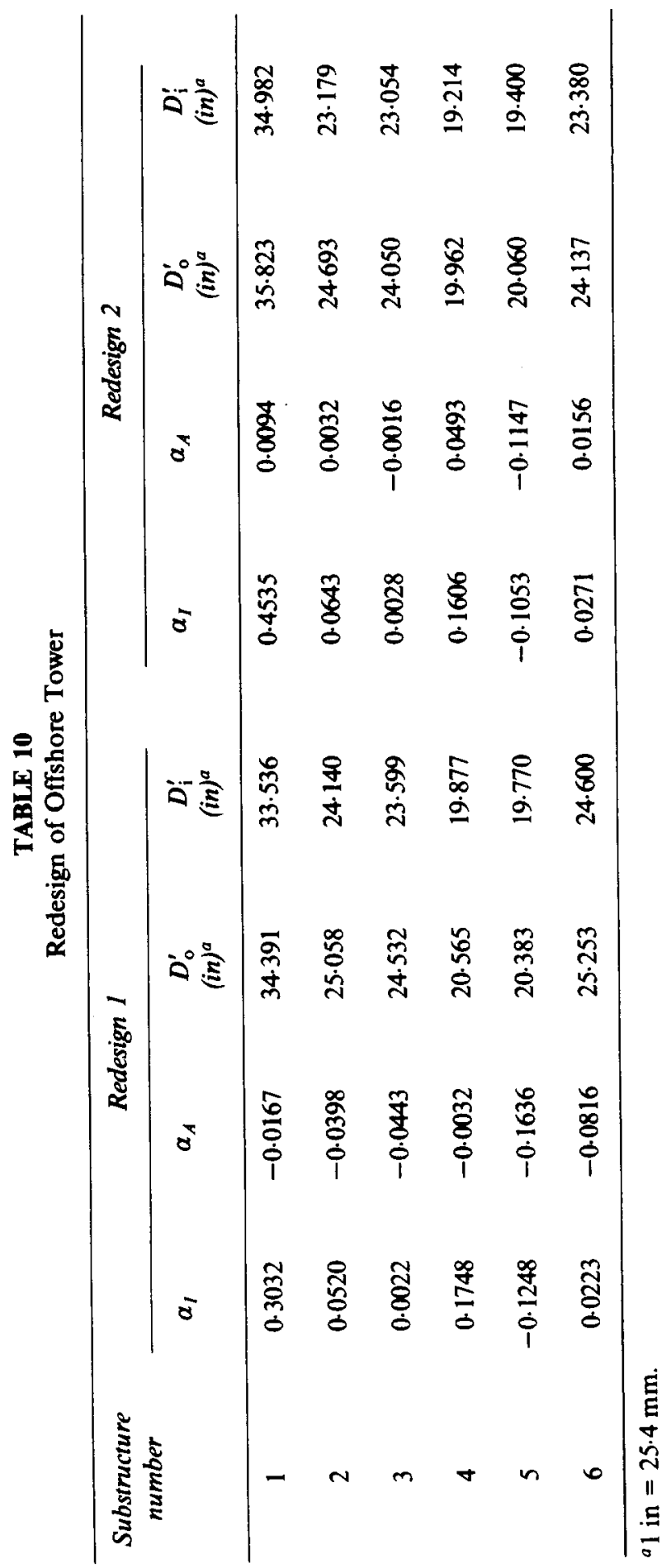




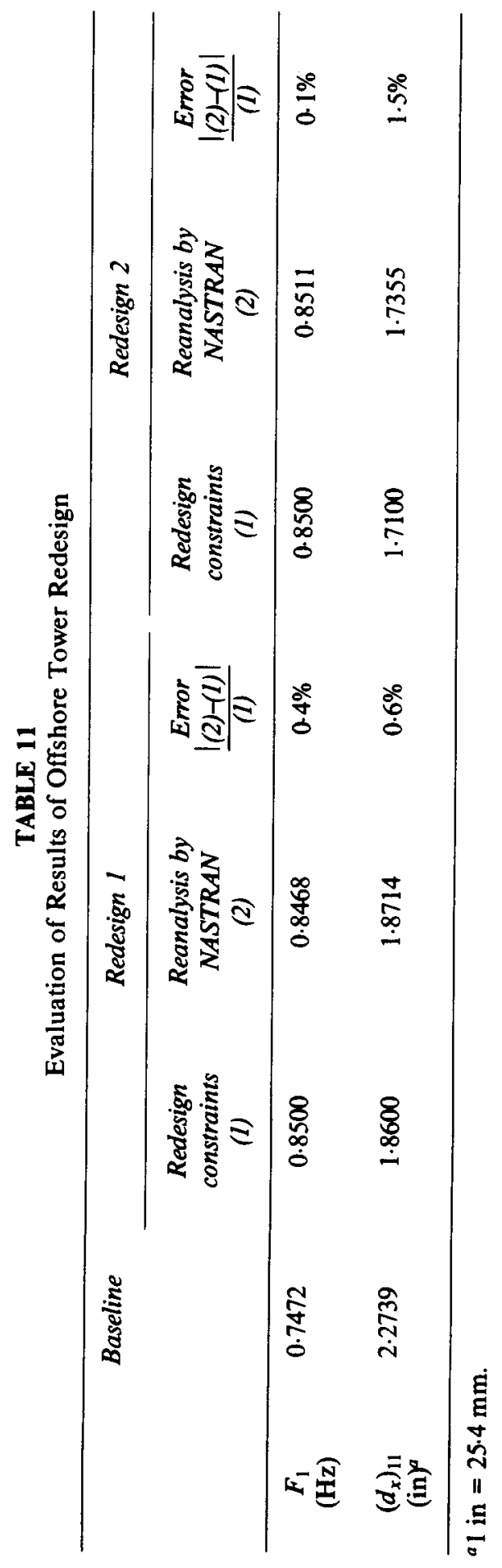


and decreasing the mass of the tower the design goals of increasing the first natural frequency and decreasing the maximum displacement are achieved. If further reduction of the tower weight is desirable, a minimum weight optimality criterion should be used in place of the minimum structural change criterion used in this application. In Redesign 2 the maximum displacement reduction requirement is stricter, resulting in higher stiffness increase and minor change to the structural mass. In both redesign problems the following data are relevant:

(i) Number of incremental steps $N=4$.

(ii) Number of redesign variables $\alpha_{e}, p=12$.

(iii) Number of used modes in modal expansions $n_{\mathrm{r}}=10$.

(iv) CPU time for static analysis running NASTRAN on IBM $3090-400$ is $5831 \mathrm{~ms}$.

(v) CPU time for dynamic analysis running NASTRAN on IBM $3090-400$ is $16565 \mathrm{~ms}$.

TABLE 12

CPU Time (ms) for Redesign of Offshore Tower, Running RESTRUCT on IBM 3090-400

\begin{tabular}{lrrrrrr}
\hline \multirow{2}{*}{ Operation } & \multicolumn{4}{c}{ Increment $N$} & \multirow{2}{*}{ Total } \\
\cline { 2 - 5 } & \multicolumn{1}{c}{1} & 2 & 3 & 4 & \\
\hline Predictor Phase & & & & & \\
(i) $\quad$ Generation of linear & & & & 507 & 2050 \\
& dynamic equations & 510 & 526 & 507 & 33 & 133 \\
(ii) $\quad$ Solution by optimization & 33 & 34 & 33 & 52582 & 211148 \\
(iii) $\quad$ Admixture coefficients & 52759 & 53271 & 52536 & 5258
\end{tabular}

Corrector Phase

(iv) Generation of nonlinear dynamic equations

(v) Generation of nonlinear static equations

(vi) Solution by optimization

Totals $\begin{array}{lllll}16112 & 16136 & 16008 & 16010 & 64266\end{array}$

$\begin{array}{lllll}7416 & 7400 & 7379 & 7340 & 29535\end{array}$

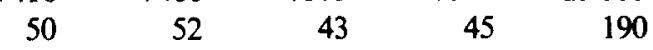

Database operations

$\begin{array}{lllll}76880 & 77419 & 76506 & 76517 & 307322\end{array}$

Miscellaneous operations

Overall total 
Table 12 shows a breakdown by operation and increment number of the CPU time required by RESTRUCT to run on IBM 3090-400. The most time consuming operation of RESTRUCT is that of computing the admixture coefficients at the end of the predictor phase in each increment. The overall redesign time is about 15 times the CPU time required for static and dynamic analysis by NASTRAN. Factors affecting the total cost of redesign by RESTRUCT are (i) the number of modes used, $n_{\mathrm{r}}$, in modal expansions, (ii) the number of incremental steps $N$, (iii) the number of redesign constraints and variables, and (iv) the number of elements in the FE model.

The offshore tower has repeated eigenvalues because its geometry and structural properties do not change by a $90^{\circ}$ rotation about the $z$-axis. As shown in Fig. 7 the $z$-axis is the axis of symmetry of the tower because it is the intersection of its two planes of symmetry. Equation (62) shows that when $\omega_{i}=\omega_{j}$, admixture coefficient $c_{i j}$ is not defined. As explained in Section $3, c_{i j}$ can be set equal to zero. Then, when the static and dynamic equations are formulated in the corrector phase, the system of these equations is first purged of non-dominant coefficients to accelerate solution.

The simple redesign beam problems studied show that RESTRUCT can be used by designers to gain experience in redesign at an affordable cost. The observations made on the redesign of the clamped-hinged beam are obvious for such a simple and small-scale structure. For largerscale structures and combinations of constraints whose effect on the objective structure is not so obvious RESTRUCT provides a unique tool for efficient redesign and identification of incompatible constraints. Finally, it should be mentioned that earlier versions of RESTRUCT have been used to redesign larger-scale structures with only static ${ }^{16}$ or only dynamic ${ }^{5.6 .11-13}$ constraints. In these earlier versions different algorithms were used which could handle only static or only dynamic constraints. The largest structure redesigned so far is a 1253-d.o.f. microcomputer disk drive $^{12}$ with one frequency constraint. The integrated static/dynamic redesign algorithm developed in this work is faster and more efficient in database operations and accuracy in addition to being able to handle both static and dynamic redesign constraints.

\section{CONCLUSIONS AND IMPACT ON STRUCTURAL DESIGN}

A direct redesign method based on perturbation has been developed and shown to be an attractive alternative to trial and error redesign 
techniques. Objectives in the design process become constraints in redesign. The algorithm developed and implemented in code RESTRUCT can handle static displacement, frequency and eigenmode constraints. RESTRUCT serves as a postprocessor to general and special purpose FE codes and can thus be used for redesign of large-scale structures. Further, it allows for large structural changes and can satisfy constraints which represent large changes in response properties. The cost of redesign depends on the number of degrees of freedom of the FE model, the number of redesign constraints, the size of the desirable changes to response particulars, the desired accuracy of the results and, most important, on compatibility of redesign constraints. The latter is obviously an important factor even in simple trial and error approaches. In any event perturbation-based redesign is conclusive - in the sense that it will produce an optimal or a minimum error design - and inexpensive compared to trial and error techniques. Several numerical applications were used to illustrate the method and look into problems of compatibility of redesign constraints, number of eigenmodes in modal expansions, repeated eigenvalues, and equality and inequality redesign constraints.

The perturbation-based redesign method developed in this work and implemented in RESTRUCT is expected to have impact on several aspects of design of marine structures. First of all, RESTRUCT being a postprocessor to general or special purpose FE programs automates redesign, reduces the required man-hours in data preparation, and takes the guesswork out of the redesign process. In addition it informs the designer of possible incompatibilities in the redesign constraints, can handle structures with repeated eigenvalues, and can produce a local optimal redesign in the vicinity of the baseline structure. Thus the designer may look forward to redesign as a means of producing a feasible design or simply improving his baseline structure within the original design constraints.

Redesign techniques have other important applications in structural design which the authors are currently pursuing. Specifically, the four following problems can be recast as redesign problems and are problems that attract significant research effort in structural reliability.

(i) Model correlation: Mathematical models imperfectly reflect reality. The error in modelling is in general unknown. Computer (numerical) models, like FE models, provide approximations to mathematical models. Thus computer models may poorly represent the physical structure and may therefore poorly predict the response of structures. This is particularly true for 
marine structures for which the manufacturing process is not very accurate. The model correlation problem is not so severe in aerospace structures or in general structural components which are manufactured more accurately.

(ii) Identification of failure mode: The number of possible modes of failure of even simple structures is too large to permit enumeration. In structural reliability this is a challenging problem that currently prohibits application of sophisticated probabilistic techniques to large scale structures like marine structures. RESTRUCT can be used to identify dominant (most probable) failure modes according to some optimality criterion like (a) minimum structural change, (b) minimum strain energy change, or (c) minimum weight change between baseline and objective structures. In this case redesign constraints are used to establish bounds of structural failure.

(iii) Structural redundancy: Large-scale structures are indeterminate to a high degree. Redundancy towards certain loads and environmental conditions is built into structures. The degree of redundancy can be computed by RESTRUCT by finding the difference between baseline and damaged structures in the process of identification of failure modes.

(iv) Redundancy in deteriorated structures: Structures age and deteriorate in time. For example corrosion and fatigue may cause failure of structural members. RESTRUCT can be used to identify failure modes and redundancy of aged or deteriorated structures. This process may be carried out sequentially till structural collapse.

\section{ACKNOWLEDGEMENTS}

This publication is a result of work sponsored by The University of Michigan/Sea Grant/Industry Consortium in Offshore Engineering under Michigan Sea Grant College Program, projects number E/GLE-14 and R/T-23, under grant number NA85AA-D-SG045C from the Office of Sea Grant, National Oceanic and Atmospheric Administration (NOAA), US Department of Commerce, and funds from the State of Michigan. Industry participants include: the American Bureau of Shipping; Conoco, Inc.; Exxon Production Research; Friede and Goldman, Ltd; Noble, Denton \& Associates, Inc.; Shell Companies Foundation (19851986); and the US Coast Guard. The US Government is authorized to produce and distribute reprints for governmental purposes notwithstanding any copyright notation appearing hereon. 


\section{REFERENCES}

1. Stetson, K A., Perturbation method of structural design relevant to holographic vibration analysis. ALAA Journal, 13 (4) (1975) 457-9.

2. Stetson, K. A. \& Palma, G. E., Inversion of first-order perturbation theory and its application to structural design. ALAA Journal, 14 (4)(1976) 454-60.

3. Stetson, K. A., Harrison, I. R. \& Palma, G. E., Redesigning structural vibration modes by inverse perturbation subject to minimal change theory. Computer Methods in Applied Mechanics and Engineering, 16 (2) (1978) 151-75.

4. Stetson, K. A. \& Harrison, I. R., Redesign of structural vibration modes by finite-element inverse perturbation. ASME Transactions, Journal of Engineering for Power, 103 (2) (1981) 319-25.

5. Sandström, R. E., Inverse perturbation methods for vibration analysis. In Proceedings, NATO Advanced Study Institute of Optimization of Distributed Parameter Structural Systems, University of Iowa, May 20-June 4, 1980.

6. Sandström, R. E. \& Anderson, W. J., Modal perturbation methods for marine structures. Transactions, SNAME, 90 (1982) 41-54.

7. Kim, K. O., Anderson, W. J. \& Sandström, R. E., Nonlinear inverse perturbation method in dynamic analysis. AlAA Joumal, 21 (9) (1983) 1310-16.

8. Kim, K. O., Nonlinear inverse perturbation method in dynamic redesign. PhD Dissertation, Department of Aerospace Engineering, The University of Michigan, Ann Arbor, MI, 1983.

9. Kim, K. O. \& Anderson, W. J., Dynamic condensation in structural dynamics redesign. In Proceedings of 7th International Symposium on the Computational Aspects of the Finite Element Method, Chicago, IL, Aug. 1983; ALAA Journal, 22 (11) (1984) 1616-17.

10. Kim, J., Integrated static/dynamic redesign of marine structures. $\mathrm{PhD}$ Dissertation, Department of Naval Architecture and Marine Engineering, The University of Michigan, Ann Arbor, MI, 1988.

11. Bernitsas, M. M., Hoff, C. J. \& Kokarakis, J. E., Nonlinear inverse perturbation in structural redesign of risers. In Proceedings of the 3rd Offshore Mechanics and Arctic Engineering Symposium, New Orleans, LA, February 12-14, 1984; ASME Transactions, Journal of Energy Resources Technology, 107 (2) (1985) 256-63.

12. Hoff, C. J., Bernitsas, M. M., Sandström, R. E. \& Anderson, W. J., Nonlinear incremental inverse perturbation method for structural redesign. In Proceedings of ALAA/ASME/ASCE/AHS 24th Structures, Structural Dynamics and Materials Conference, Lake Tahoe, Nevada, May 1983; ALAA Journal, 22 (9) (1984) 1304-9.

13. Hoff, C. J. \& Bernitsas, M. M., Nonlinear inverse perturbation methods in structural dyna mics redesign of offshore structures. In Proceedings of the 3rd International Congress on Marine Technology, Athens '84, Athens, Greece, May 28-June 1, 1984.

14. Hoff, C. J. \& Bernitsas, M. M., Dynamic redesign of marine structures. Journal of Ship Research, 29 (4) (1985) 285-95.

15. Hoff, C. J., Static/dynamic redesign of marine structures. PhD Dissertation, Department of Naval Architecture and Marine Engineering, The University of Michigan, Ann Arbor, MI, 1985. 
16. Hoff, C. J. \& Bernitsas, M. M., Static redesign of offshore structures. In Proceedings, 5th International OMAE Symposium, Tokyo, Japan, 1986, Vol. III, pp. 78-85.

17. Bernitsas, M. M. \& Kim, J., RESTRUCT: a program for redesign of structures. Report to The University of Michigan/Sea Grant/Industry Consortium in Offshore Engineering; Publication No. 303, Department of Naval Architecture and Marine Engineering, The University of Michigan, Ann Arbor, MI, Sept. 1987.

18. Gans, H. D., Structural optimization including centrifugal and Coriolis effects. PhD Dissertation, Department of Aerospace Engineering, The University of Michigan, Ann Arbor, MI, 1987.

19. Haftka, R. T. \& Prasad, B., Programs for analysis and resizing of complex structures. Computers and Structures, 10 (1979) 323-30.

20. Choi, K. K., Haug, E. J. \& Seong, H. G., An iterative method for finite dimensional structural optimization problems with repeated eigenvalues. International Journal for Numerical Methods in Engineering. 19 (1983) 93112.

21. Haug, E. J., Choi, K. K. \& Komkov, V., Design Sensitivity Analysis of Structural Systems. Academic Press, New York, 1986.

22. Luenberger, D. G., Introduction to Linear and Nonlinear Programming. Addison-Wesley, Reading, MA, 1973.

23. Bradley, S. P., Hax, A. C. \& Magnanti, T. L., Applied Mathematical Programming. Addison-Wesley, Reading, MA, 1977.

24. Gill, P. E., Murray, W., Saunders, M. A. \& Wright, M. H., User's guide for SOL/QPSOL: a Fortran package for quadratic programming. Department of Operations Research, Stanford University, CA, 1983.

25. Shanno, D. F. \& Phua, K. H., Algorithm 500, minimization of unconstrained multivariate functions. Association for Computing Machinery, Transactions on Mathematical Software, 6 (Dec. 1980) 618-22.

26. Fox, R. L., Optimization Methods for Engineering Design. Addison-Wesley, Reading, MA, 1971.

27. Gill, P. E., Murray, W., Saunders, M. A. \& Wright, M. H., User's guide for SOL/NPSOL: a Fortran package for nonlinear programming. Department of Operations Research, Stanford University, CA, 1983.

28. Strang, G., Linear Algebra and its Applications. Academic Press, New York, 1986. 